Jens Soentgen

\title{
Argumentieren mit Nichtwissen: Die Risikodiskurse über Mobilfunk und Grüne Gentechnik
}

\section{Einleitung}

Die Risikodiskurse über Grüne Gentechnik und Mobilfunk waren Gegenstand zahlreicher soziologischer Untersuchungen. Auch die Argumente, die in diesen Diskursen ausgetauscht werden, sind vielfach untersucht worden. ${ }^{1}$ Im Folgenden soll ausgehend von dem empirischen Material, das in unserem Projekt „Nichtwissenskulturen“ anhand von insgesamt 69 Interviews erhoben wurde, ${ }^{2}$ versucht werden, mithilfe eines prominenten Arguments, des argumentum ad ignorantiam, ein Modell zu konstruieren, das einen Überblick über den Diskurs ermöglicht. Dies wird in der Literatur meist anhand der Akteure, anhand der historischen Entwicklung oder anhand der Streitfragen vermittelt. Hier wird nun ein neuer Weg beschritten, indem ausgehend von dem genannten Argument ein an logischen $\mathrm{Zu}$ sammenhängen, und nicht an kontingenten Konstellationen, orientierter Überblick versucht wird.

Dieses Modell eröffnet, und dies zeichnet den hier gewählten Zugang gegenüber anderen aus, zugleich einen Möglichkeitsraum, indem es nicht nur gestattet, jene Argumente einzuordnen, die auch tatsächlich, nach Maßgabe unserer Untersuchung, im Diskurs geäußert werden, sondern zugleich auch Argumente erkennen lässt, die möglich wären, obgleich sie im Verlauf unserer Interviews nicht geäußert wurden. Schließlich gestattet die Rekonstruktion, die erhebliche Bedeutung der unterschiedlichen Nichtwissenskulturen in den untersuchten Diskursen genauer zu spezifizieren (vgl. dazu ausführlicher Kastenhofer in diesem Band). Insgesamt versucht mein

1 Stellvertretend für viele seien genannt: Lassen/Jamison (2006); Cook (2004); Wiedemann et al. (2001: bes. 52-86; 2005); van den Daele (2001); van den Daele et al. (1997).

2 Aus Platzgründen verzichte ich bei der folgenden Darstellung, von wenigen Ausnahmen abgesehen, auf Zitate aus unseren Interviews. Gleichwohl achte ich darauf, tatsächlich geäußerte Positionen stets klar erkennbar zu halten und sie von möglichen, aber im empirischen Material nicht formulierten Argumenten zu unterscheiden. 
Beitrag, die von einzelnen Autoren (z. B. Keith/Rehg 2008) geforderte, aber nur sehr selten vollzogene Verbindung von argumentativen Analysen und Science Studies zu realisieren. Ein Angebot, ausgehend von dem Schema eine Abwägung der Argumente vorzunehmen, wird abschließend formuliert.

Das argumentum ad ignorantiam, das seit der Antike nachgewiesenermaßen in Gebrauch ist, ${ }^{3}$ schließt, kurz gesagt, vom Nichtfinden (bzw. Nichtwissen) von etwas auf dessen (und sei es nur: wahrscheinliches) Nichtvorhandensein. Ein offensichtlich prekärer und angreifbarer Schluss, der mit ,Widersprüchen schwanger geht ${ }^{6}$, wie man in der Tradition Hegels sagen würde. Hinter dem Argument steht die Einsicht in die prinzipielle Unmöglichkeit, einen möglichen Schaden ausschließen zu können, wie intensiv auch immer geforscht wird. Der an sich einfache Satz „Diese Technologie ist unschädlich" kann nicht bewiesen werden. Allein Schäden lassen sich eindeutig nachweisen, Unschädlichkeit aber nicht. Der Risikodiskurs ist nicht schließbar. Irgendwann muss man dennoch zu einem Ergebnis kommen - und an dieser Stelle taucht das Argument auf.

Es ist ein schwaches Argument, aber gerade das macht es so interessant. Wird dieses Argument in einer Diskussion vorgebracht, so kann der Gegner in seiner Antwort die internen Probleme dieses Arguments thematisieren. Das kann auf unterschiedliche Weisen geschehen, aber nicht auf beliebige Art und Weise. Das Argument eröffnet einen bestimmten Spielraum von Reaktionsmöglichkeiten, der inzwischen, vor allem dank der theoretischen Arbeit des kanadischen Philosophen und Argumentationstheoretikers Douglas N. Walton (1996), ${ }^{4}$ recht gut untersucht ist. Es lohnt sich, von dem Diskussionsstand der Forschung zu diesem Argument zu

3 Vgl. etwa eine Bemerkung, mit der Plinius Berichte von „Magiern“ kommentiert, wonach der Salamander ein Tier sei, das Feuer löschen könne: ,si forent vera, iam esset experta roma“: „Wenn diese Berichte wahr wären, wäre der Sachverhalt in Rom längst bekannt." Den Schluss, dass die Berichte eben falsch sind, weil der Sachverhalt nicht zum konsolidierten römischen Wissen gehört, darf der Leser selbst ziehen, womit sich das Argument als Enthymem, also als rhetorischer Schluss erweist, der nicht vollständig ausgeführt ist, um den Eindruck von Pedanterie zu vermeiden (vgl. Plinius Secundus: Naturalis Historia, liber xxix, XXIV (manchmal auch XXIII).

4 Zum Hintergrund und Kontext der Arbeit Waltons vgl. van Eemeren und Grootendorst (2004, insb. Kap. 7). Hinweisen möchte ich auch auf Arbeiten von Gerd Gigerenzer, der das Argument, ohne es explizit zu bezeichnen und ohne Bezug zu den Untersuchungen, die diesem in der Philosophie und Argumentationstheorie gewidmet wurden, im Kontext seiner Studien zur „Rekognitionsheuristik” diskutiert (vgl. etwa Gigerenzer 2007: 117-144). 
lernen und diesen Diskussionsstand für das Verständnis der Risikodiskurse über Mobilfunk und Grüne Gentechnik fruchtbar zu machen.

Warum gerade dieses Argument? Beim Stichwort „Nichtwissensargument" könnte man auch an anderes denken, zum Beispiel an die Überlegung: „Man weiß nicht, ob es schädlich ist, lassen wir es also lieber“. Auch dieses Enthymem, von dem, wie jeder weiß, viele volkstümliche Varianten im Umlauf sind („Wat de Bur nich kennt, dat frett he nich“, wie man beispielsweise am Niederrhein sagt), spielt eine bedeutende Rolle beim Umgang von Menschen mit Risiken und unbekannten Situationen. Es bietet Menschen Orientierung in risikoreichen Situationen, insbesondere bei der Ernährung: Unbekannte Speisen werden stehengelassen, die Schwelle, Neues, Unbekanntes zu probieren ist nahezu überall sehr hoch. Erst wenn man sich durch vorsichtiges Probieren kleiner und kleinster Mengen, durch Beobachtung folgenlosen Verzehrs durch andere usw. mit der Speise vertraut gemacht hat, wird sie eventuell genossen. Dieses Verhalten ist zweifellos sinnvoll, es ist emotional tief verankert und findet sich auch bei Tieren. Das Verhaltensmuster, das dem Argument entspricht, wird unter dem Stichwort „Neophobie“ erforscht (Jones 1990: 40 und passim).

Auch über die Ernährung hinaus ist dieses Verhalten zweifellos bedeutend und spielt ebenso bei Risikodiskursen stets eine beträchtliche Rolle. Bei gesellschaftlichen Auseinandersetzungen in einer freiheitlichen, demokratischen und innovationsfreudigen Gesellschaft ist das Argument der mangelnden Kenntnis nicht, wie in traditionalen Gesellschaften, Grund genug, die neue Technik oder Praktik zu unterlassen, sondern Ausgangspunkt für Forschung und Erprobung. Man sagt: „Wir kennen es nicht, es könnte schädlich sein, untersuchen wir es also näher." Dies ist der Ausgangspunkt der Sicherheitsforschung, wie sie auch zur Grünen Gentechnik und zum Mobilfunk stattgefunden hat und, in inzwischen vermindertem Umfang, weiterhin stattfindet. Auch sie aber führt nicht zu Unbedenklichkeitsgarantien. Zum einen kann das Argument auf höheren Stufen, wenn schon eingehende Beschäftigung mit möglichen Risiken stattgefunden hat, wiederholt werden; stets kann man sagen: „Wir wissen einfach noch nicht genug.“ (vgl. hierzu die Aussagen von der Warte komplexitätsorientierter Nichtwissenskulturen im Beitrag von Kastenhofer in diesem Band). Zum anderen führt Sicherheitsforschung selbst für den, der der Überzeugung ist, dass ,nichts' gefunden wurde, höchstens zu jener Feststellung, die ich als argumentum ad ignorantiam bezeichnet habe. Jetzt hört man: „Gäbe es Schäden, hätten wir sie finden müssen. Wir haben aber nichts gefunden. Also ..." 
Das argumentum ad ignorantiam taucht somit erst in einer Spätphase von Risikodiskursen auf, weil es einiges voraussetzt. Auch dieses Argument ist aber, zumindest in den hier diskutierten Diskursen, kein Abschluss, sondern eher ein Zwischenfazit, das eine neue Phase der Auseinandersetzung einleitet, um die es hier geht. Im reifen Stadium von Sicherheits- und Technikfolgenforschungsdiskursen, das heißt, in dem Stadium, in dem bereits einige Risikoforschung stattgefunden hat, spielt das argumentum ad ignorantiam oft eine bedeutende Rolle, und insofern ist es sinnvoll, es auch für eine Strukturierung des Diskurses einzusetzen.

Öffentliche Diskurse wurden bislang meistens und durchaus erfolgreich mit soziologischem Vokabular untersucht; hier soll nun, wie gesagt, der Versuch gemacht werden, rhetorische beziehungsweise dialektische ${ }^{5} \mathrm{Be}-$ griffe und Forschungsresultate fruchtbar zu machen, was an sich naheliegend ist, jedoch, wenn man von der ganz einseitigen Konjunktur der Metapher absieht, noch allzu selten geschieht. ${ }^{6}$

Natürlich können konkrete Diskurse nicht aus argumentationstheoretischen Überlegungen abgeleitet werden. Sie wuchern in viele Richtungen. Interessant ist aber, dass die formalen und verallgemeinerten Studien zur Struktur des Arguments auf Hauptlinien der Argumentation verweisen, die auch in dem empirischen Material auffindbar waren. Deshalb liefert eine Betrachtung des argumentum ad ignorantiam so etwas wie einen Schlüssel für die Risikodiskurse über Grüne Gentechnik und Mobilfunk. Zumindest lassen sich die Argumente, die in unseren Interviews geäußert wurden, und die wir nicht ohne Grund als repräsentativ ansehen für die jeweiligen Diskurse, mithilfe dieses Arguments in übersichtlicher Form darstellen.

Entsprechend gehe ich im Folgenden zunächst auf das Argument selbst ein, charakterisiere das empirische Material und werde dann die Risikodiskurse von diesem Argument her darstellen. Es wird sich zeigen, dass die Argumentationsstruktur wie auch die Argumentationsdynamik mit diesem Hilfsmittel verständlicher werden. Ein Vergleich der beiden Diskurse und eine Prognose über ihren weiteren Verlauf runden die Darstellung ab.

5 Für Aristoteles ist die Dialektik die Kunst der Disputation und die Rhetorik ein Teilgebiet der Dialektik (Aristoteles, Topik: 27; Rhetorik: 1. und 2. Kapitel). Die neuere Literatur orientiert sich wieder an der aristotelischen Einteilung (und nicht am platonischen oder gar hegelianischen Dialektikbegriff; siehe besonders: van Eemeren/Grootendorst 2004).

6 Sogar unter dem expliziten Titel einer Argumentativen Diskursanalyse findet man in einem neueren Handbuch der Diskursanalyse vor allem eine Metaphernanalyse (Hajer 2003: 271-298). 


\section{Allgemeine Charakterisierung und Geschichte des argumentum ad ignorantiam}

Gegen Ende des Kapitels „On Reason“ seines Werkes „An Essay Concerning Human Understanding“ (zuerst 1690) zählt John Locke vier Argumente auf, ,die die Menschen in ihrer Beweisführung anderen gegenüber meist verwenden, um sie für ihre Ansicht zu gewinnen oder sie wenigstens so einzuschüchtern, dass ihr Widerspruch zum Schweigen gebracht wird“ (Locke 1962/1690: 389). ${ }^{7}$ Zu diesen vieren zählt auch der folgende Argumentationstyp: „Ein zweites Mittel, das man häufig anwendet, um andern zuzusetzen und sie zu zwingen, ihre Meinung preiszugeben und sich der bestrittenen Ansicht anzuschließen, besteht darin, dass man dem Gegner zumutet, er solle entweder das, was man als Beweis anführt, anerkennen, oder aber einen besseren Beweis erbringen“" (ebd.: 389). ${ }^{8}$ Diese Form des Arguments tauft Locke auf den Namen argumentum ad ignorantiam, ${ }^{9}$ und es scheint, dass er diese Bezeichnung geprägt hat, zumindest konnte ich sie in der älteren rhetorischen Literatur nicht auffinden, obwohl das Argument selbst bereits in der Antike gebraucht wurde.

Die Kennzeichnung Lockes ist allgemeiner sowie weitaus ungenauer als das, was in der modernen dialektischen und rhetorischen Literatur unter dem Titel argumentum ad ignorantiam untersucht wird; es ist nicht einmal auszuschließen, dass er einen ganz anderen Schluss im Sinn hatte. Jedenfalls wurde seine prägnante neulateinische Bezeichnung übernommen. Das Argument wird heute vor allem in der angelsächsischen Logikliteratur dargestellt und untersucht, meist wird es dabei als Fehlschluss

7 Originaltext: ,that man in their reasoning with others, do ordinarily make use of to prevail on their assent, or at least so to awe them as to silence their opposition".

8 Originaltext: „Another way that men ordinarily use to drive others and force them to submit their judgments and receive the opinion in debate is to require the adversary to admit what they allege as a proof or to assign a better. "

9 Weiter erwähnt Locke das argumentum ad verecundiam, ad hominem und ad judicium. Ad verecundiam (verecundia $=$ Ehrfurcht): gemeint ist der Versuch, andere durch Benennung von Experten oder anderen Autoritäten zu beeindrucken; ad iudicium (iudicium = Urteil, Einsicht): wenn jemand, schreibt Locke, auf der Grundlage des Wissens oder der Wahrscheinlichkeit argumentiert, solche Argumente sind laut Locke die besten, da sie wirklich belehren; ad hominem: bei John Locke ist damit gemeint, dass man einen Gegner mit Aussagen angeht, die von ihm selbst stammen. Vgl. auch die vier „Weisen, die Gewinnung eines Schlusses zu verhindern“, welche Aristoteles gegen Ende des achten Buches seiner „Topik“ darstellt (Aristoteles Topik: 192; 8. Buch, 10. Kapitel, Bekker 161a). 
betrachtet. ${ }^{10}$ Der kanadische Philosoph Douglas N. Walton hat demgegenüber in mehreren Publikationen, deren wichtigste in seinem Werk „Arguments from Ignorance“ (1996) zusammengefasst sind, nachgewiesen, dass es vom Kontext abhängt, ob solche Argumente tatsächlich Fehlschlüsse (bzw. untriftige Argumente) sind oder nicht. Dabei weist er nach, dass das Argument nicht nur in Risikodiskursen (er führt die Diskussion über Risiken von Brustimplantaten aus Silikon an), sondern auch zum Beispiel in Diskussionen über die Existenz von Geistern oder Außerirdischen, in Hexenprozessen ebenso wie in manchen politischen Debatten eine zentrale Rolle einnimmt. Walton gibt dem Argument, im Anschluss an die umfangreiche Literatur, ${ }^{11}$ die folgende Form:

„If A were true, it would be known (proved, presumed) to be true.

A is not known (proved, presumed) to be true.

Therefore, A is (presumably) false." (Walton 1996: 277)

Dabei bezeichnet A einen bestimmten Sachverhalt. Die von Walton gewählte Umschreibung weicht, wie oben gesagt, nicht unerheblich von der Locke'schen Vorlage ab; sie entspricht indessen dem, was seither in der Logikliteratur unter dem Titel dieses Arguments verhandelt wird. Die etwas umständliche Formulierung verfremdet das Argument - und verdeckt, dass es sich um ein überaus häufiges, geradezu alltägliches Argument handelt. Es ist in abgekürzter, gerundeter Form geradezu redensartlich, besonders in dem Ausruf „Das wüsste ich aber!“, mit dem alltäglich bestimmte Behauptungen gekontert werden: „Der Sowieso soll der bedeutendste lebende Philosoph sein!? - Das wüsste ich aber!" Hier wird aus dem eigenen Nichtwissen auf das Nichtsein (bzw. auf die Unwahrheit der Behauptung) geschlossen. Es handelt sich also um ein Argument, welches gerade aus dem Nichtwissen und dem Nichtkennen von etwas noch eine

10 Siehe für viele das im angelsächsischen Sprachraum sehr verbreitete Logiklehrbuch von Irving M. Copi: „Introduction to Logic“ (Copi 1968: hier bes. 63f.). Formal ist das Argument korrekt, es handelt sich um einen Modus tollens; strittig ist lediglich der Inhalt der zwei Prämissen. Ein Überblick über die historische Entwicklung findet sich bei Hansen und Pinto (1995); siehe dort auch den Artikel von Erik C. W. Krabbe (1995: 251-264).

11 Walton widmet das zweite Kapitel seines Buches dem historischen Rückblick (vgl. Walton 1996: 24-63). Rätselhaft ist, weshalb er in diese Darstellung nicht auch Leibniz einbezieht, der doch bekanntlich unter dem Titel „Nouvaux Essais“ eine eingehende und detaillierte Auseinandersetzung mit Locke formuliert hat. Gerade das Kapitel „Über die Vernunft“, in dem Leibniz auch auf das von Locke getaufte argumentum ad ignorantiam mit lesenswerten Bemerkungen eingeht, ist für die Geschichte der Logik von höchster Bedeutung, weil Leibniz hier seine Erweiterung der Aristotelischen Syllogismen darlegt. 
Information bezieht. Da die Situation des Nichtwissens und Nichtkennens von etwas aber so etwas wie die allgemeine conditio humana ist, handelt es sich um ein verbreitetes und zentrales Argumentationsmuster. ${ }^{12}$ Jeder kennt dieses Argument und jeder verwendet es.

Das Besondere an dieser Gedankenfigur ist, dass hier aus dem Nichtwissen ein Gewinn, eine Sicherheit gezogen wird, was freilich nur unter ganz bestimmten Voraussetzungen und in ganz bestimmten Kontexten überzeugend möglich ist. Nichtwissen wird so gewendet, dass es nicht nur Anlass zu Verlegenheit ist, sondern zur produktiven Kraft wird. In Situationen sehr fragmentarischer Evidenz, wie sie nicht nur in Risikodiskursen, sondern auch zum Beispiel beim Studium antiker Quellen regelmäßig vorkommen, ist das argumentum ad ignorantiam eine routinisierte Praktik, um auf sehr spärlicher Grundlage noch handlungsleitende Informationen folgern zu können. So ist es bei Althistorikern etwa üblich, aus dem Schweigen eines Textes über ein bestimmtes Ereignis, das dem Autor hätte bekannt sein müssen, und das für seinen Text relevant gewesen wäre, zu folgern, dass der Text vor diesem Ereignis entstanden sein muss. Man spricht dann vom argumentum e silentio. Mit seiner Hilfe werden sehr oft antike und historische Texte datiert.

Was geschieht nun mit dem Argument, wenn es im Anschluss und mit Verweis auf wissenschaftliche Risikoforschung vorgebracht wird? Sorgt es für klare Verhältnisse? Der Schriftsteller Jules Verne, dessen Wissenschaftsverständnis mehrere Generationen von Europäern prägte, konnte noch einen seiner Protagonisten sagen lassen: „Assez. Quand la science a prononcé, il n’y a plus qu'à se taire.“ („Genug. Wenn die Wissenschaft gesprochen hat, muss man schweigen."; Verne 1864, Voyage au centre de la terre, xiv: 84, zit. nach: Martin 1985: 160) Doch eine solche Haltung wurde im 20. Jahrhundert zunehmend brüchig, im 21. Jahrhundert ist sie nur noch von antiquarischem Interesse. ${ }^{13}$ Tatsächlich schließt das Argument nur selten wirklich einen Diskurs ab, sondern läutet eher eine neue Phase desselben ein - denn es kann offensichtlich bestritten werden. Walton schließt aus seinen Fallstudien, dass es auf ein solches Argument zwei Reaktionen gibt:

12 Gerd Gigerenzer identifiziert daher genau dieses Argument mit der von ihm so genannten Rekognitionsheuristik (vgl. Gigerenzer 2007: 29-49 und passim).

13 Gregor Schiemann hat die Entwicklung der wissenschaftlichen Aussagen im Theoriebereich als zunehmende Hypothetisierung gekennzeichnet, eine offensichtlich parallele Erscheinung (vgl. Schiemann 1997). 
Jens Soentgen

„1. If A were true, would it be known (proved, presumed) to be true?

2. Is it the case, that A is not known (proved, presumed) to be true?"

(Walton 1996: 277) ${ }^{14}$

In beiden Fragen wird die Wissensgrundlage, auf deren Basis das Argument vorgetragen wird, infrage gestellt, und zwar in zwei Richtungen: zum einen, ob sie wirklich vollständig oder zumindest ausreichend ist, und zweitens, ob es sich wirklich um Wissen handelt (und nicht zum Beispiel um vorschnelle Verallgemeinerungen, Irrtümer oder gar Fälschungen), beziehungsweise ob das Wissen richtig interpretiert wird. In den Fragen werden die schwachen Punkte des Arguments zur Sprache gebracht. Denn es ist offenkundig, dass das Argument inhaltlich keineswegs zwingend ist und Widerspruch in einer ganz bestimmten Weise geradezu herausfordert. Es kann ein anmaßendes, bigottes Argument sein, wenn jemand sein eigenes Wissen zum Maß von Sein und Nichtsein macht und das, was er selbst nicht kennt, für inexistent erklärt.

Das Argument kann andererseits auch sehr überzeugend sein, wenn es sich um ein gut abgrenzbares Thema handelt. Oder auch, wenn dahinter eine gewaltige, über mehrere Generationen fortgesetzte, kollektive Anstrengung steht, etwas Bestimmtes in Erfahrung zu bringen.

So hat die Physik zum Beispiel die Tatsache, dass bislang trotz zahlreicher Versuche kein funktionierendes Perpetuum mobile erster Art erfunden werden konnte, ein System also, das Energie aus nichts erzeugt, den Satz abgeleitet, dass ein solches generell unmöglich ist. Dieser Satz ist heute der erste Hauptsatz der Thermodynamik. Der zweite Hauptsatz der Thermodynamik besagt, dass es auch kein Perpetuum mobile zweiter Art gibt, ein System also, das Wärme ohne Temperaturgefälle in Arbeit umwandelt.

\section{Die Funktion des argumentum ad ignorantiam in Risikodiskursen}

Für meinen Zweck, die Nutzung des Arguments für eine Analyse der Argumentationsstruktur und -dynamik der Risikodiskurse über Grüne Gentechnik und Mobilfunk, möchte ich nun sowohl die Darstellung des Arguments selbst als auch die Formulierung der Sätze, mit denen es infrage

14 In einer erneuten, zehn Jahre später veröffentlichten zusammenfassenden Darstellung (Walton 2006: 321-326) nennt er folgende zwei Gegenfragen: „How complete is the search for knowledge found in the argumentation?" und „How complete does the knowledge need to be to support the argument adequately?" 
gestellt werden kann, weiterentwickeln und um neue Aspekte ergänzen. In beiden Diskursen geht es um die Frage, ob diese Technologien gesundheitliche und/oder ökologische Schäden verursachen. Dieser Frage wurden und werden zahlreiche Studien gewidmet. Die Befürworter der jeweiligen Technologien formulieren nun das argumentum ad ignorantiam etwa so:

Wenn es Schäden gäbe, hätte man sie mittlerweile in den Studien gefunden.

Man hat aber keine Schäden gefunden.

Also kann man (bis auf Weiteres) davon ausgehen, dass die Technologien unschädlich sind.

Dieses Argument ist nicht etwa eine unter vielen Möglichkeiten, aus einem negativen Ergebnis gründlicher Sicherheitsforschung einen Schluss zu ziehen, sondern die einzig mögliche. Würde man nämlich nicht erwarten, dass durch Sicherheitsforschung („Studien“) Probleme eher gefunden werden als ohne, dann könnte man sich diese Forschung sparen. Wäre es von vornherein illegitim, von einem negativen Studienergebnis auf eine, sei es auch eingeschränkte Unbedenklichkeit zu schließen, bräuchte man ebenfalls keine Sicherheitsforschung zu betreiben; dann wäre ein präventives Verbieten aller neuen Produkte anzudenken. Gleichwohl kann das Argument natürlich bestritten werden.

Gegner oder Skeptiker knüpfen an das Argument Fragen, die im Folgenden nacheinander behandelt werden:

(1) Waren die bisherigen Untersuchungen ausreichend? Walton hatte ausgeführt, dass es beim argumentum ad ignorantiam unter anderem von der Struktur der zugrunde liegenden ,knowledge base“ ${ }^{15}$ abhängt, ob es ein triftiges Argument ist oder ein unplausibles. So konnte er zeigen, dass das argumentum ad ignorantiam unter anderem dann fast zwingend ist, wenn der Bereich der Suche beschränkt oder legitim beschränkbar ist. ${ }^{16}$

15 Direkt übersetzt: Wissensbasis. Man könnte etwas freier auch mit „Erfahrungsraum" übersetzen und so einen Begriff nutzen, den Peter Wehling für die soziologische Nichtwissensforschung fruchtbar gemacht hat (vgl. Wehling 2006: hier bes. 24f.). Der Ausdruck „Erfahrungsraum“ wurde von Reinhart Koselleck geprägt, der auch darauf hinwies, dass jeder Erfahrungsraum durch einen Erwartungshorizont geprägt sei (vgl. Koselleck 1995).

16 Man kann den Suchraum auf einer Skala anordnen, je nachdem, ob er mehr oder weniger begrenzt und lokal ist. Der größtmögliche Suchraum ist der Kosmos, und tatsächlich tauchen argumenta ad ignorantiam immer wieder, auch in sehr ausgearbeiteter Form, in astronomischen Debatten auf, etwa in denen der Bioastronomie (Suche nach außerirdischem Leben). Schon in der „Encyclopaedia of Ignorance“ (Duncan/Weston-Smith 1977) beschäftigten sich die meisten Artikel mit astronomischen Themen, was zweifellos mit der Unendlichkeit dieses Erfahrungsraumes 
In der Risiko- und Sicherheitsforschung geht es nun sehr oft darum, ob eine bestimmte Technologie oder ein bestimmter Stoff neben den erwünschten auch unerwünschte Effekte hat, typischerweise negative Effekte auf die Gesundheit. Das klingt simpel, wenn man sich vorstellt, dass man einfach den Faktor $\mathrm{X}$ an einer hinreichend großen Zahl von Versuchspersonen oder Versuchstieren testen müsse, um feststellen zu können, ob dieser negative gesundheitliche Effekte hervorbringe oder nicht. Das Problem, das sich in ähnlicher Weise allen kausalanalytischen Untersuchungen stellt, liegt aber darin, dass viele Wirkungen, insbesondere gesundheitliche Wirkungen nicht von einer Ursache abhängen, sondern von bestimmten Kombinationen von Ursachen. Wenn daher ein Prüffaktor keine Wirkung zeigt, muss dies nicht heißen, dass er generell kausal irrelevant ist. Vielleicht wirkt er nur in Kombination mit bestimmten anderen Faktoren, zum Beispiel mit bestimmten Vorerkrankungen. Theoretisch ist daher die Aufgabe nahezu jeder Sicherheitsforschung unendlich, da nicht nur einzelne Faktoren, sondern auch alle denkbaren Kombinationen mit anderen Faktoren zu testen wären. Hier liegt auch ein wichtiger Grund, weshalb ein negatives Ergebnis nicht der Beweis der Unschädlichkeit ist: Denn es kann sein und ist auch sehr oft der Fall, dass der geprüfte Faktor nur in Kombination mit anderen Faktoren oder aber nur bei Abwesenheit eines gewöhnlich vorhandenen, hemmenden Faktors die Wirkung hervorbringt. Daher rührt die Asymmetrie, dass man zwar aus positiven Tests, wenn diese korrekt durchgeführt wurden, auf kausale Relevanz, aber aus negativen Tests nicht ohne Weiteres auf kausale Irrelevanz schließen kann (vgl. für eine moderne, formalisierte Darstellung dieses Problems kausalen Schließens Baumgartner/Graßhoff 2004: 213f.).

Viele Stoffe entfalten schädliche Wirkungen nur dann, wenn ganz bestimmte organische oder genetische Faktoren hinzukommen. Die unendliche Vielfalt der potenziell relevanten Faktorenkombinationen lässt sich in einer realen Risikoforschung, sofern sie nicht über unendlich viel Zeit und unendliche Mittel verfügt, nicht abarbeiten. Auch sehr aufwendige Sicherheitsforschung kann immer nur die nächstliegenden Kombinationen in den Blick nehmen. So kommt es, dass auch bei gründlich getesteten Technologien nach einiger Zeit negative Wirkungen bekannt werden können, die trotz intensiver Sicherheitsforschung übersehen wurden und die durchaus so gravierend sein können, dass die Produktion gestoppt werden muss. Ein

zusammenhängt, aber auch damit, dass wir ihn, wie John Kendrew in seiner Einleitung zu Band 2 der Enzyklopädie bemerkte, nur sehr eingeschränkt erkunden können (Kendrew 1977: 206f.). 
besonders plastisches Beispiel ist Thalidomid, das in dem Schlafmittel Contergan, das von 1957 bis Ende 1961 vermarktet wurde, enthalten war. Es bewirkt Fehlbildungen bei menschlichen Föten, nicht aber bei üblichen Labortieren. Andererseits sind auch positive Wirkungen möglich und kommen vor. Auch hierfür ist Thalidomid ein Beispiel, denn es wirkt, wie sich 1964 herausstellte, gegen Lepra.

Die Unmöglichkeit, den Prüffaktor mit allen relevanten Ko-Faktoren zu kombinieren, die möglicherweise gemeinsam unerwünschte Wirkungen hervorbringen können, ist nur eines der grundlegenden Probleme jeglicher Sicherheitsforschung. Ein anderes ist die Unmöglichkeit, in Studien die gesamte Zeit abzudecken, in der Wirkungen möglich sind. Sicherheitsstudien haben einen Horizont von Monaten bis Jahren, Langzeiteffekte können aber auch erst nach Jahrzehnten auftreten. Ein weiteres Problem liegt in bestimmten Methoden, etwa dem Einsatz von Versuchstieren. Resultate aus Tierversuchen sind nicht ohne Weiteres auf den Menschen übertragbar, wie wiederum das Beispiel Thalidomid zeigt, bei dem zwar die sedierende Wirkung an Labormäusen entdeckt werden konnte, nicht aber die fruchtschädigende Wirkung, da sich diese bei Labormäusen nicht entfaltet. Insgesamt ist Risiko- und Sicherheitsforschung stets ein Kompromiss zwischen inhaltlichen Zielsetzungen und organisatorischen, zeitlichen, methodischen sowie finanziellen Limitationen. Dies lässt immer wieder die kritische Rückfrage zu, ob denn die Untersuchungen ausreichend waren.

(2) Stimmt es, dass wirklich nichts gefunden wurde? In nahezu allen Diskursen, die sich an ein argumentum ad ignorantiam knüpfen, werden Ergebnisse kontrovers diskutiert. Schon die Frage, ob etwas gefunden wurde, kann kontrovers sein, wenn Studien nicht reproduzierbar sind. Noch umstrittener ist dann die Interpretation der Ergebnisse, bei der oft Unterscheidungen, etwa zwischen Schaden und ,bloßem' Effekt einerseits, zwischen Nachweisen und Hinweisen andererseits wichtig sind. ${ }^{17}$

17 Beim Thema Unterscheidungen denken viele deutsche Leser zuallererst an Niklas Luhmann, der sich seit den 1980er Jahren, ausgehend von George Spencer Browns Werk „Laws of Form“ (1972), aber natürlich auch mit deutlichen und manchmal expliziten Anknüpfungen an Hegels Logik, eingehend mit Unterscheidungen und ihrer Bedeutung vor allem für die Operationen gesellschaftlicher Funktionssysteme, jedoch auch mit ihrer Bedeutung für die Theorie befasste. Schon in den fünfziger Jahren hatte sich auch der Logiker und Rhetoriktheoretiker Chaïm Perelman mit Unterscheidungen befasst, auf deren Bedeutung für konkrete Argumentationen er in seinem Werk „Traité de l'Argumentation - La Nouvelle Rhétorique“ (1958, mit Lucie Olbrechts-Tyteca) ausführlich eingeht. Ich halte mich im Folgenden ausschließlich an Perelman, dessen Bemerkungen auf einer ähnlichen Linie wie Luhmanns Ausführungen liegen, aber näher an konkreten Argumentationen gearbeitet 
Unterscheidungen dienen nicht nur der Einteilung, sondern haben auch einen argumentativen Sinn. Sie entstehen, wie Perelman betonte, in Situationen des Dissenses, wenn eine Mehrzahl von Stimmen sich widerspruchsvoll äußert. In dieser Situation hilft die Unterscheidung von Hinweisen und Nachweisen. Man kann dann sagen: Es gibt Hinweise, aber keine wirklichen Nachweise. Eine aporetische Situation im Diskurs ist damit überwunden. Sie ist sozusagen ,aufgehoben', der Meinungsaustausch kann nun, anknüpfend an diese Unterscheidung, weitergehen. Natürlich ist der Dissens damit nicht verschwunden, aber er befindet sich nun sozusagen auf einem anderen Niveau. Die Blockade ist aufgehoben, man kann weiterforschen, weitere Informationen sammeln, weil man nun nachsehen kann, ob sich die ,Hinweise' zu ,Nachweisen' verdichten lassen, was in der vorherigen Situation, in der einfacher Widerspruch gewissermaßen lähmte, nicht möglich war. Zugleich entsteht neuer Streit, denn nun muss diskutiert werden, wie genau zwischen Nachweisen und Hinweisen unterschieden wird und ob es nicht noch weitere Stufen zwischen beiden gibt (Kastenhofer 2007). Ein allgemeines ,Rezept', wie Hinweise in Nachweise überführt werden könnten, gibt es dabei nicht. Dennoch führt dieser Streit zu neuen Informationen und ist daher fruchtbar.

Insofern ist also diese Unterscheidung eine Art Instrument, das einen starren, unfruchtbaren Streit in einen flüssigen und beweglichen verwandelt. Sie verwandelt also nicht Dissens in Konsens, sondern ist ein künstliches Hilfsmittel, um einem Dissens, der sich festgefahren hat, einen neuen Anknüpfungspunkt zu geben. Dementsprechend sind solche Unterscheidungen streng genommen auch keine Argumente, da sie isoliert nicht dazu dienen, die Zustimmung zu einer These zu verstärken. Sie sind auch mehr als bloß Topoi, Fundstätten für Argumente. Vor allem sind sie semantische Hilfsmittel, die versuchen, einen Diskurs an einer bestimmten Stelle wieder ,flottzumachen'.

(3) Sind die Umstände so beschaffen, dass wir diesen Stand der Dinge akzeptieren sollten, auch wenn er keine abschließende Sicherheit bietet? Dies ist eine normative Frage, die in gewisser Weise außerhalb des Arguments selbst liegt. Möglicherweise ist sie aus diesem Grund nicht von Walton aufgeführt. Sie ist aber deshalb zu berücksichtigen, weil im konkreten Diskurs sehr oft von der sachlichen auf die normative Ebene gewechselt wird. Dies ist aufgrund der Aporien des Arguments auch notwendig, bedenkt man die Unmöglichkeit, in endlicher Zeit und mit endli-

und daher für meine Zwecke brauchbarer sind (vgl. Perelman/Olbrechts-Tyteca 1999/1958). 
chen Mitteln definitiv über die Unbedenklichkeit neuer Technologien urteilen zu müssen. So kann ein Technologiebefürworter, der der Ansicht ist, dass genug geforscht sei, behaupten: „Wir verlieren kostbare Zeit, wenn wir uns auf weitere exzessive Sicherheitsforschung einlassen. Deutschland verliert den Anschluss in einem zentralen Technologiebereich der $\mathrm{Zu}-$ kunft!“ Worauf ein Gegner kontern könnte: „Die befürchteten Schäden sind so groß, dass es zu gefährlich wäre, die Technologie beim derzeitigen Wissensstand umzusetzen." Wie kann auf die Frage reagiert werden? Man kann den Stand der Dinge zum Beispiel akzeptieren, wenn das erwartete Schadensausma $\beta$ bei Nichthandeln geringer ist als das erwartete Schadensausmaß bei Handeln, oder auch, wenn das Handeln reversibel ist, also alles zurückgerufen werden kann, sollten doch Probleme auftreten.

So weit diese auf den Arbeiten von Walton aufbauende Darstellung der Rückfragen, die jemand stellen kann, dem das argumentum ad ignorantiam vorgelegt wird. Diese drei Fragen scheinen hinreichend zu sein, um den Diskurs zu strukturieren. Es gibt natürlich noch die Option, das Argument grundsätzlich zu bestreiten und etwa zu sagen: „Es reicht nicht, dass keine Schäden gefunden wurden, es muss auch die Unschädlichkeit nachgewiesen werden." Jedoch führt diese Stellungnahme in einen toten Bereich, sie lässt dem Partner keinen Ausweg - denn es ist kaum möglich, die Unschädlichkeit von irgendetwas definitiv zu beweisen. Natürlich handelt es sich um eine mögliche Stellungnahme, dennoch bringt sie den Diskurs nicht weiter, sondern würgt ihn ab, indem sie dem Partner eine nicht erfüllbare Beweislast aufbürdet. Insofern kann die Option in der weiteren Diskussion außer Betracht bleiben, auch wenn gelegentlich solche Ansichten geäußert werden. Es scheint mir, dass es außer den genannten keine weiteren, grundsätzlich anderen Möglichkeiten gibt, das Argument kritisch infrage zu stellen.

Nun zu den zwei Risikodiskursen. In beiden taucht das Argument, wenn auch nur in abgekürzter Weise (als Enthymem), immer wieder auf, in der Regel auf der Seite derjenigen, die den Gebrauch dieser Technik befürworten und der Ansicht sind, dass die durchgeführten Studien keine Hinweise auf mögliche Gefährdungen geben. Man versucht, aus vorangehenden Bemühungen eine verbindliche Schlussfolgerung zu ziehen. $\mathrm{Zu}-$ gleich fasst das Argument die bisherigen Stimmen gewissermaßen zusammen und versucht, ein Fazit zu ziehen ${ }^{18}$ - und gerade an dieses Fazit

18 Daher taucht das Argument typischerweise auch in den Resümees von Studien auf, siehe zum Beispiel die Formulierungen der vier Metagutachter in Wiedemann et al. (2003: 10-12). 
knüpfen sich natürlich wieder viele widerstrebende Stimmen. Der Diskurs wird übersichtlich, wenn man ihn sozusagen durch die Brille dieses Arguments betrachtet. Es ist also eine Form der Beobachtung dieser Diskurse, die sinnvoll ist: Die Stimmen des Diskurses lassen sich übersichtlich arrangieren, wenn man von diesem Argument ausgeht.

\section{Zwei Risikodiskurse im Lichte des argumentum ad ignorantiam}

Auf die Einzelheiten der beiden Technologien muss und kann an dieser Stelle nicht eingegangen werden. Nur auf einige Besonderheiten des Diskurses, die vor allem im internationalen Vergleich von Bedeutung sind, sei hingewiesen. Sowohl die Diskussion des Mobilfunks als auch die Diskussion der Grünen Gentechnik finden in Deutschland vor dem Hintergrund der EU-Gesetzgebung statt und sind von dieser maßgeblich geprägt. $\mathrm{Zu}-$ nächst einige Hinweise zum Diskurs über Grüne Gentechnik (siehe hierzu auch Böschen in diesem Band). Rund 80 Prozent aller Deutschen und 65 Prozent aller Europäer lehnen Grüne Gentechnik ab. Seit 1998 bestand in der EU deshalb ein Moratorium für die Einfuhr und Zulassung von gentechnisch veränderten Organismen, im Fachjargon GVOs (oder englisch GMOs). Eine Klage der USA vor der Welthandelsorganisation WTO auf Schadenersatz für entgangene Gewinne führte zur Kennzeichnungsverordnung der EU, die im April 2004 in Kraft trat.

Der Anbau bestimmter geprüfter gentechnisch veränderter Produkte ist in Europa (mit Ausnahme der Schweiz, in der seit 2005 ein Moratorium gilt) damit grundsätzlich zugelassen. Ausgenommen von dieser Kennzeichnungspflicht sind Fleisch, Eier, Milchprodukte, welche von Tieren stammen, die mit gentechnisch veränderten Stoffen gefüttert wurden. Auch Produktzusätze (z. B. Labferment, das mithilfe gentechnisch veränderter Bakterien hergestellt wurde, sowie Enzyme, z. B. in Waschmitteln), Zusatzstoffe und Aromen, die von gentechnisch veränderten Mikroorganismen hergestellt wurden, sind von der Regelung ausgenommen, da sie nicht als Lebensmittel gelten. Zudem gilt die Kennzeichnungspflicht generell nur, wenn die Verunreinigung mit genetisch verändertem Material über 0,9 Prozent liegt.

Um die Koexistenz von ökologischem Anbau und Landwirtschaft mit GVOs zu ermöglichen, hat sich die EU bereits auf Schwellenwerte und Abstandsregelungen beim Anbau verständigt. Denn einige Nutzpflanzen vermehren sich durch Pollenflug. Wenn also ein Landwirt gentechnisch verändertes Saatgut ausbringt und sein Nachbar eine Ernte einfährt, die 
mit gentechnisch veränderten Pflanzen ungewollt verunreinigt wird, dann muss der Genbauer haften und entschädigen. Der Schwellenwert für die Verunreinigung wurde auf 0,9 Prozent festgelegt.

Nun noch einige Hinweise zum Mobilfunk. ${ }^{19}$ Die ersten Mobiltelefone in Deutschland nutzte 1918 die Deutsche Reichsbahn, doch erst 1958 gab es das erste Mobilfunknetz in Deutschland, das sogenannte A-Netz. Ein Bund ein C-Netz folgten, inzwischen nutzt man die digitalen D- und ENetze. Erst in den 1990er Jahren kam es zur massenhaften Verbreitung von Mobiltelefonen, seither gibt es auch einen öffentlichen Risikodiskurs. Für die spezifische Absorptionsrate, den sogenannten SAR-Wert, gilt in Deutschland derzeit der Grenzwert von 2 Watt $/ \mathrm{kg}$ biologischem Gewebe.

Unter dem Diskurs über Grüne Gentechnik beziehungsweise über Mobilfunk verstehe ich die Gesamtheit aller Stimmen, die öffentlich zu Risiken dieser Technologien Stellung beziehen. Wer auch immer sich öffentlich zu einer dieser Technologien äußert, und sich dabei implizit oder explizit auf andere Meinungen zum Thema bezieht, bucht sich in den Diskurs ein. Dies ist, verglichen mit den Diskursbegriffen etwa von Foucault oder auch von Habermas, ein schwacher Diskursbegriff, der aber für die Zwecke dieser Studie brauchbar ist, weil er die Aufmerksamkeit sogleich auf die Argumente lenkt.

Wie kann man diesen Diskurs analysieren? Zum einen kann man versuchen, die zu dem Diskurs gehörenden Stimmen in den bekannten öffentlichen Arenen aufzusuchen und herauszufiltern. In unserem Projekt wurden von Anfang 2004 bis Mitte 2006 insgesamt 30 Experten zur Grünen Gentechnik befragt und 25 Experten zum Thema Mobilfunk; hinzu kamen vierzehn Vorgespräche. Die Interviewpartner wurden bewusst aus unterschiedlichen Kontexten ausgewählt, aus Wissenschaft, Wirtschaft, NGOs, Politik und Behörden; die Interviews waren leitfadengestützt; die Aussagen der Interviewpartner wurden anonymisiert.

Die Interviews zeichnen einzelne, an dem Diskurs beteiligte Stimmen auf. Um nachzuweisen, dass es tatsächlich Stimmen sind, die zum Diskurs gehören, und nicht lediglich persönliche Meinungen von Privatpersonen, war es nicht nötig, auf die Intention der Sprecher zurückzugehen. Vielmehr ließ sich dies in jedem Fall an einer formalen Eigenheit der Äußerungen zeigen: In vielen Sequenzen äußerten sich die Interviewpartner nämlich nicht nur direkt zu den Fragen der Interviewerin, sondern antworteten zugleich auf Stimmen, die zuvor in diesem Diskurs geäußert wurden, kommentierten frühere Diskussionsbeiträge, polemisierten versteckt wie

19 Die historischen Bemerkungen entnehme ich Grasberger/Kotteder (2003: 22f.). 
offen oder parodierten andere Ansichten. Die Interviewten sprachen gewissermaßen nicht nur zu der anwesenden Interviewpartnerin, sondern ebenso auch zu unsichtbaren, abwesenden Anderen, Gegnern oder Befürwortern, die in diesem Diskurs zugleich mit ihnen unterwegs waren oder sind. In jeder der von uns befragten Stimmen waren deutliche Echos und Resonanzen auf andere Stimmen vernehmbar, nicht nur in einzelnen Aussagen, sondern teils sogar noch in den kleinsten Einheiten der Aussagen: Bis in die Wortwahl waren manche Bemerkungen dialogisiert. Die Stimmen des Diskurses hören einander ständig und rufen sich Antworten zu.

Typisch für diese Spiegelungen ist folgendes Zitat aus einem unserer Interviews:
„Zunächst wurde gesagt: ,Raps kreuzt nicht aus.' Dann schauen wir uns die Lite- ratur an: Die OECD stellt 1997 fest, und es gibt kilometerweise Literatur: Raps kreuzt aus. Schade drum. Nächstes Argument, die Blühfenster zu den Kreuzungs- partnern überschneiden sich ja gar nicht. Raps blüht im Mai, die Kreuzungspartner im August. Dann kann ja nichts passieren. Schauen wir nach: Raps außerhalb von Kulturflächen blüht im Prinzip das ganze Jahr über und nicht nur zu den Zeiten, wo kultivierter Raps blüht. Nächstes Argument: Ja, aber die kommen ja gar nicht in denselben Gebieten vor. Da gab es jeweils dann keine Information dazu. Wir machen eine Untersuchung dazu und stellen fest: Natürlich kommen sie ganz her- vorragend in denselben Gebieten vor." (INT 5) ${ }^{20}$

Der Interviewpartner hätte auch sagen können: „Raps kreuzt aus, er blüht zur selben Zeit wie seine Kreuzungspartner und kommt auch in denselben Gebieten vor." Mit diesen Aussagen hätte er sich unmittelbar auf den Sachverhalt bezogen. Aber sein Argument ist doppelt ausgerichtet, nicht nur auf den Sachverhalt, sondern auch auf Behauptungen der Gegenseite. Die Replik reagiert gespannt auf eine unsichtbare Position, indem sie ihr antwortet beziehungsweise sie antizipiert. ${ }^{21}$ Viele Passagen unserer Interviews kommen mit einem solchen expliziten oder impliziten Seitenblick auf die Positionen der Gegenseite daher und erweisen sich gerade dadurch schon in ihrer Struktur als Beiträge zu einem Diskurs. Es ist klar, dass die Inhalte sich von dieser Struktur nicht trennen lassen; alles, was sich an Inhalten ausfindig machen lässt, verdankt sich ja dem Streit der Stimmen.

20 Ich beziehe mich hier auf die sequenzierten Interviews, die in unserer Datenbank vorliegen.

21 Die Rhetorik solcher doppelt gerichteter Aussagen ist ausführlich und besonders plastisch von Michail Bachtin (der russische Name wird auch „Bakhtin“ transkribiert) dargestellt worden, insbesondere im fünften Kapitel seines Werkes „Probleme der Poetik Dostoevskijs“ (Bachtin 1971/1963: 202-228). 
Der Diskurs begegnet uns zunächst als eine Polyphonie von Stimmen, als ein Labyrinth von Benennungen, Thesen, von Ansichten und Standpunkten, die sich in vielfältiger Weise aufeinander beziehen, einander hervorlocken und sich aneinander ausformen. Jedes Argument, jede Meinung darin kann dargestellt werden als Replik auf zuvor geäußerte Argumente und Meinungen, als ein gebrochenes Echo auf andere, zuvor oder gleichzeitig (oder sogar zukünftig) geäußerte Standpunkte. Die Interviews sind in den Diskurs eingebucht und zugleich eine verdichtete Probe dieses Diskurses. Die wesentlichen Argumente und Standpunkte, welche in den Diskursen bislang geäußert wurden, finden sich auch in den Interviews.

An dieser Stelle noch eine Anmerkung zu dem hier verwandten Begriff des Arguments. Argumente sind in Diskursen wesentliche Elemente, denn ihre Funktion ist, die Zustimmung zu bestimmten Meinungen, die in dem Diskurs geäußert werden, zu schwächen oder zu stärken. Ein Argument ist also nicht einfach eine beliebige Äußerung, sondern ein sprachlicher Akt, der sich an ein Publikum wendet, mit dem Ziel, dieses von der Gültigkeit oder Ungültigkeit einer bestimmten Meinung zu überzeugen. Argumentieren ist also zunächst ein soziales Tun. Die Formen, in denen dies geschehen kann, sind sehr vielfältig und keineswegs auf die Schlussmuster der formalen Logik beschränkt. Schon Aristoteles unterschied in seiner Topik 200 bis 300 verschiedene Muster, wie Argumente gearbeitet sein können. Die bis heute in der Literatur unterschiedenen Muster für Argumente wurden bislang nie komplett gesammelt, sie dürften allerdings eher eine fünfstellige als eine vierstellige Zahl umfassen.

Wie kann man nun aber aus den Interviews die Struktur des Diskurses rekonstruieren? Eine Option ist es, die Argumente herauszufiltern, die von den Interviewpartnern benannt wurden. Wir haben das mithilfe des Programms Max QDA durchgeführt und das Thema jedes Arguments mit einem Stichwort benannt. Dabei zeigte sich, dass die meisten Stichworte jeweils Stoff sowohl für ein Pro- als auch für ein Kontra-Argument hergaben, dass es sich also um Topoi handelt. Tatsächlich kommt es in dem Diskurs zu Paarbildungen von Argumenten. Kein Argument bleibt lange alleine stehen, schon bald wächst ihm ein Widerpart zu. Ina Rust konnte in jedem der beiden Diskurse rund 60 solcher Argumentpaare rekonstruieren und in jeweils einer Liste arrangieren, wobei die am häufigsten genannten Argumente die oberen Listenplätze einnehmen (vgl. Rust 2006a, 2006b). Die Listen enthalten wesentliche Punkte und Ansichten, die in den beiden untersuchten Diskursen von Anfang 2004 bis Mitte 2006 geäußert wurden. Sie beinhalten gleichwohl nicht alle Meinungen und Ansichten des jeweiligen Diskurses - auch wenn mit zunehmender Zahl der Interviewpartner 
die Wiederholungen immer mehr zunahmen, also die zusätzlichen Interviews immer weniger neue Argumente zutage förderten, so kamen in jedem neuen Interview doch auch immer wieder einzelne überraschende Ansichten zum Ausdruck. Das letzte Wort ist eben in keiner dieser Kontroversen bereits gesprochen worden.

Gleichwohl gibt die hohe Rate an Wiederholungen Anlass zu begründeter Zuversicht, dass das von uns eingefangene Meinungsbild nicht nur ein willkürlicher Schnappschuss ist, sondern wesentliche Punkte des Diskurses enthält. Anhand der vorliegenden Literatur wurde ebenfalls nochmals kontrolliert, ob wesentliche Punkte hier enthalten sind. Die zwei von Ina Rust erstellten Listen repräsentieren eine Möglichkeit des Arrangements der Stimmen. Sie erlauben einen bedeutenden Lerneffekt - sie regen nämlich dazu an, von dem einseitigen Blick auf nur eine Seite loszukommen und immer auch die Gegenargumente mitzuhören. Ein Nachteil dieses Arrangements ist allerdings, dass eine innere Struktur, die über die Paarbildung hinausgeht, auf diese Weise nicht sichtbar wird.

Ich möchte daher im Folgenden eine Rekonstruktion des Diskurses vorschlagen, die eben von dem oben besprochenen Nichtwissensargument ausgeht. Die Rekonstruktion ist keine Tabelle, sondern ein Schema, das genauso gebaut ist wie der Diskurs selbst: als ein Wechselspiel von Fragen und Antworten, als eine Folge von - oftmals zerdehnten - Wortwechseln. Die Stimmen werden aufgeteilt in befürwortende Stimmen und Gegenstimmen („Befürworter" und „Gegner"), auch wenn das eine Vereinfachung des Beobachters ist. Das Nichtwissensargument steht jeweils am Anfang, und zwar in der folgenden, bereits dargestellten Form:

Wenn es Schäden gäbe, hätte man sie mittlerweile in den Studien gefunden.

Man hat aber keine Schäden gefunden.

Also kann man (bis auf Weiteres) davon ausgehen, dass die Technologien unschädlich sind.

An dieses Argument knüpfen sich nun folgende Fragen:

- (1) Waren die bisherigen Untersuchungen ausreichend? (mit der Unterfrage 1': Gibt es systematische Gründe, weshalb die Studien - so, wie sie betrieben wurden - gar keine Schäden nachweisen konnten?)

- (2) Stimmt es wirklich, dass keine Schäden entdeckt wurden?

- (3) Sind die Umstände so beschaffen, dass wir diesen Stand der Dinge akzeptieren sollten, auch wenn er keine abschließende Sicherheit bietet? 
An dem Modalverb sollte der dritten Frage erkennt man, dass es hier nicht mehr nur um eine Wissens- beziehungsweise Nichtwissensfrage geht. Es wird der Kontext einbezogen, aber zugleich auch eine Abwägung verlangt.

Wenn man nun das Argument und die Gegenfragen präzisiert und erweitert, erhält man eine Sequenz, welche den Streit, der im Risikodiskurs Mobilfunk vor sich geht, überraschend übersichtlich wiedergibt. Natürlich handelt es sich nicht um den Diskurs selbst, sondern um eine Beobachtung des Diskurses:

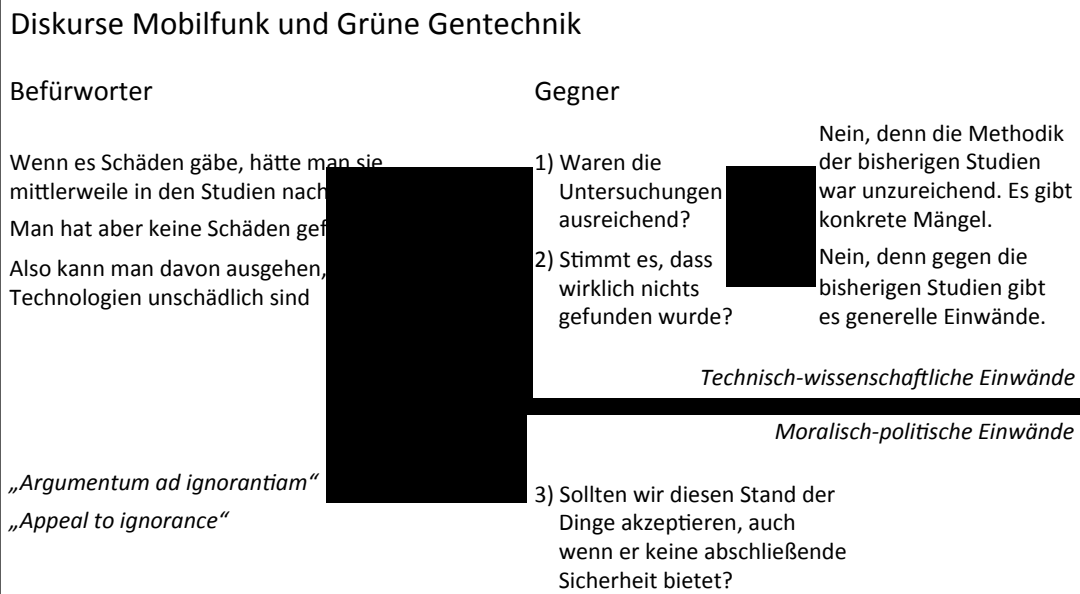

2) Stimmt es, dass Nein, denn gegen die wirklich nichts bisherigen Studien gibt gefunden wurde? es generelle Einwände.

Technisch-wissenschaftliche Einwände

Moralisch-politische Einwände

3) Sollten wir diesen Stand der Dinge akzeptieren, auch wenn er keine abschließende Sicherheit bietet?

Abbildung 1: Das argumentum ad ignorantiam als Schlüssel zu den Risikodiskursen Mobilfunk und Grüne Gentechnik

(Quelle: Eigene Darstellung)

Im ,oberen Teil' lässt sich eine Dynamik erkennen, die schon von Walton benannt wurde. $\mathrm{Zu}$ den inhaltlichen Aporien des Arguments zählt ja, wie wir bereits festgestellt hatten, die Schwierigkeit, in endlicher Zeit und mit endlichen Mitteln einen potenziell unendlichen Suchraum, zum Beispiel eine unendliche Zahl von Faktorenkombinationen, abzuarbeiten.

Ist dagegen der Suchraum irgendwie plausibel schließbar, dann ist das argumentum ad ignorantiam nahezu zwingend. Es ist daher kein Wunder, dass die Technologie-Befürworter immer wieder die Tendenz erkennen lassen, den Suchraum zu schließen und/oder nur ganz bestimmte Suchmethoden zuzulassen. Dazu gibt es verschiedene Strategien. Zum einen kann man versuchen, zu vermitteln, dass man an allen ,Orten', an denen es Sinn macht, auch nachgesehen habe - und mit allen Instrumenten und Methoden, die bei einer Suche zweckmäßigerweise eingesetzt werden. Das setzt natürlich voraus, dass andere Methoden diskreditiert werden müssen. 
Demgegenüber weisen die Gegner darauf hin, dass bestimmte ,Orte' bislang ignoriert wurden, dass mit den falschen Methoden oder gar mit ungeeigneten Instrumenten gesucht wurde, dass bestimmte relevante Faktoren gar nicht betrachtet wurden. Oft wird auch darauf hingewiesen, dass immer wieder bei technologischen Innovationen unerwartete Effekte und Schäden auftreten, mit denen niemand gerechnet hat, dass also der Erfahrungsraum durch einen zu engen Erwartungshorizont eingeschnürt sei.

Befürworter und Gegner können in diesem Wortwechsel dabei nicht auf Wissenschaft und Nicht-Wissenschaft verteilt werden.

Zudem agieren Vertreter der Wissenschaft hier keineswegs einheitlich wie man erwarten könnte. ${ }^{22}$ Vielmehr ergab unsere Untersuchung, dass in diesem Teil der Debatte ein deutlich erkennbarer Riss durch die Wissenschaft selbst ging. Auf der einen Seite fanden wir insbesondere Laborwissenschaftler, zum Beispiel Molekularbiologen, auf der anderen Seite Feldwissenschaftler (Ökologen), welche die Übertragung der Ergebnisse von Laborversuchen ins Freiland mit Skepsis verfolgten und auf viele unkontrollierbare kausale Faktoren im Freiland hinwiesen. Im Diskurs über Mobilfunk ergab sich eine ähnliche Zweiteilung zwischen Feldwissenschaften und Laborwissenschaften. Hier entsprachen den Ökologen die niedergelassenen Ärzte und Umweltmediziner (vgl. Böschen et al. 2010).

Die Beurteilung der Triftigkeit des argumentum ad ignorantiam hängt also eng mit den von uns identifizierten Nichtwissenskulturen zusammen. Dabei werden die Prämissen des Arguments nach unterschiedlichen Kriterien bewertet, die mit der Heterogenität von Nichtwissenskulturen (vgl. Wehling in diesem Band, Kap. 5) zusammenhängen, aber auch mit den unterschiedlichen Strategien zur Verknüpfung von Expertenmeinungen und epistemischen Kulturen (vgl. Kastenhofer in diesem Band, Kap. 5-7).

Eine zweite Methode, die Suche schließbar zu machen und zugleich den Schluss des argumentum ad ignorantiam zu stützen, besteht darin, ein allgemeines $\mathrm{Ma}$ für eine gründliche Suche herzunehmen. Hier gibt es verschiedene Möglichkeiten. Zum einen kann man auf die lange Zeit verweisen, welche die Suche bislang andauert. Zum anderen kann man erklären, dass in dem Diskurs schon seit Längerem keine neuen Argumente aufgetaucht seien. Schließlich kann man auch darauf hinweisen, dass diese potenziellen Risikoträger schon sehr viel intensiver auf mögliche Gefahren abgeklopft worden seien als anderes, mit denen die Kritiker tagtäglich hantieren. So kontern Gentechnik-Befürworter zum Beispiel gern, indem sie darauf hinweisen, dass Lebensmittel, die GVOs enthalten, inzwischen

22 Und wie auch formuliert wurde, siehe nur Cook (2004: 30-44). 
genauer hinsichtlich möglicher gesundheitlicher Schäden untersucht seien als herkömmliche oder gar ökologische Lebensmittel. Freilich werden die Kritiker auch hier nicht um naheliegende Antworten verlegen sein. Sie können darauf hinweisen, dass herkömmliche Lebensmittel schon seit vielen Generationen ,im Test' sind, und damit den Zeitfaktor ins Spiel bringen. Denn zu den grundsätzlichen, bereits oben dargestellten Problemen jeder Risikoforschung zählt neben der Unmöglichkeit, alle denkbaren Kombinationen kausaler Faktoren zu prüfen, dass sie zeitlich meist auf wenige Jahre befristet ist, wobei aufgrund der langen Karenzzeit vieler Schäden eher Jahrzehnte angemessen wären. Eine Risikoforschung, die auf zwei Generationen, also 60 Jahre, angelegt wäre, wäre zwar inhaltlich sehr überzeugend, jedoch unbezahlbar und käme im Effekt einem Innovationsverbot gleich. $\mathrm{Zu}$ den unauflösbaren Aporien jeder Sicherheitsforschung zählt die Unmöglichkeit, die Zeithorizonte des Manifestwerdens möglicher Risiken mit den Zeithorizonten wissenschaftlicher und wirtschaftlicher Organisationen zu versöhnen.

Deutlich wird in jedem Fall, dass sich bei Fragen wie derjenigen nach möglichen Gesundheitsrisiken einer neuen Technologie in aller Regel der Suchraum nicht überzeugend schließen lässt. Daher sind auch etwaige Hoffnungen, von der Wissenschaft ein abschließendes Wort in solchen Diskursen zu hören, auf Sand gebaut. Wenn auch die Hoffnung, dass die Wissenschaft ein für alle Male eine abschließende Aussage über Risiken des Mobilfunks treffen könnte, zumindest kurzfristig enttäuscht werden muss, so haben die Studien dennoch wichtige Details zutage gefördert, die sehr wohl erheblichen Orientierungswert haben. So geben sie Hinweise darauf, wie die Strahlungsexposition durch individuelles Nutzungsverhalten gemindert werden kann. Zugleich geben sie auch Hinweise auf technische Alternativen. Etwa könnte die Strahlungsexposition durch die Basisstationen gesenkt werden, wenn statt dreier Anbieter, die in Deutschland jeweils ein Netz aufgebaut haben und entsprechende Basisstationen unterhalten, nur ein einziger zugelassen wäre.

\section{Rekonstruktion des Risikodiskurses Mobilfunk}

Nun gehe ich auf die beiden Risikodiskurse Mobilfunk und Grüne Gentechnik ein, wobei ich als Leitfaden der Darstellung die Fragen wähle, die man an das Argument stellen kann. Hier zunächst ein Überblick: 


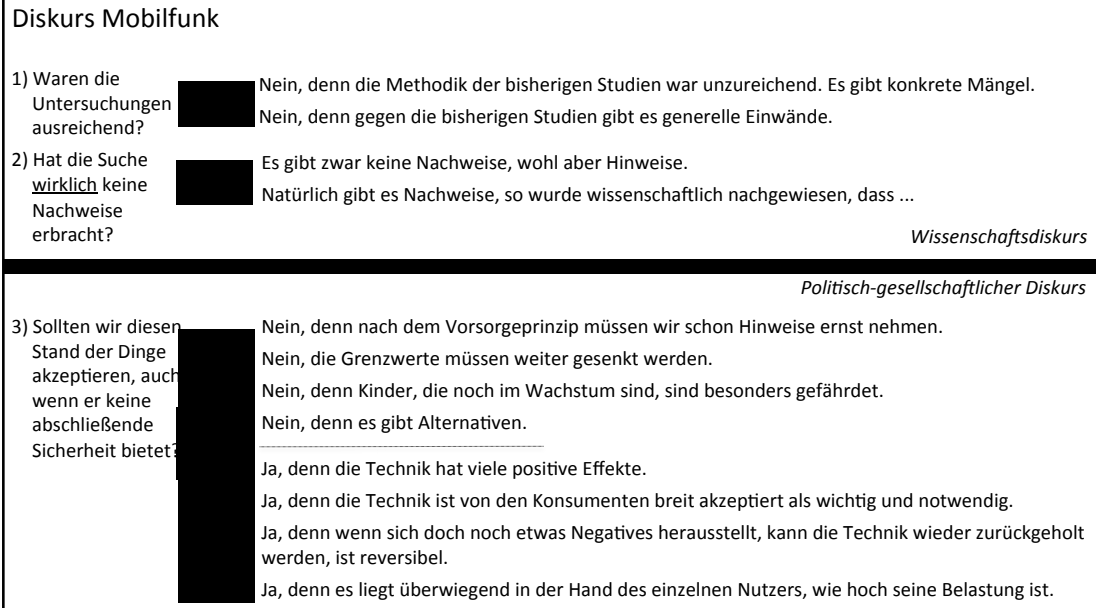

Abbildung 2: Der Wortwechsel im Mobilfunkdiskurs

(Quelle: Eigene Darstellung)

Die mit „Nein“ beginnenden Stimmen sind Stimmen der Gegner, die mit „Ja“ beginnenden Sätze sind Argumente der Befürworter. Deutlich ist, dass das Bild zweigeteilt ist - oben findet man die wissenschaftlichtechnischen Argumente und unten die ethisch-politischen. Im ,oberen' Bereich wird um Wissen und Nichtwissen gerungen, im ,unteren' Bereich geht es um Werte beziehungsweise Interessen. Beides ist freilich nicht lupenrein voneinander zu trennen.

(1) Waren die bisherigen Studien ausreichend? Auf diese Fragen antworten Befürworter und Gegner im Mobilfunkdiskurs, wie zu erwarten, unterschiedlich. Bei den Befürwortern sind die erwähnten Versuche einer Schließung unverkennbar, die Gegner sind um eine Öffnung bemüht, wie die beiden folgenden Grafiken, welche die erfassten Argumente auflisten, erkennen lassen (siehe Abbildungen 3 und 4).

Sichtbar ist, dass eine Einigung wohl schwierig ist - auch wenn die Befürworter plausible Gründe darlegen können, weshalb die bisherige Untersuchung ausreichend war, können die Gegner auf immer neue Erfahrungsräume verweisen. Hier kommen dann der Faktor Geld und auch der Faktor Zeit ins Spiel: Je intensiver Forschung wird, desto teurer gerät sie. Ein Zusammenhang, auf den besonders Nicholas Rescher immer wieder hingewiesen hat (vgl. Rescher 1999, siehe auch die neuere Behandlung des Themas in Rescher 2009). 


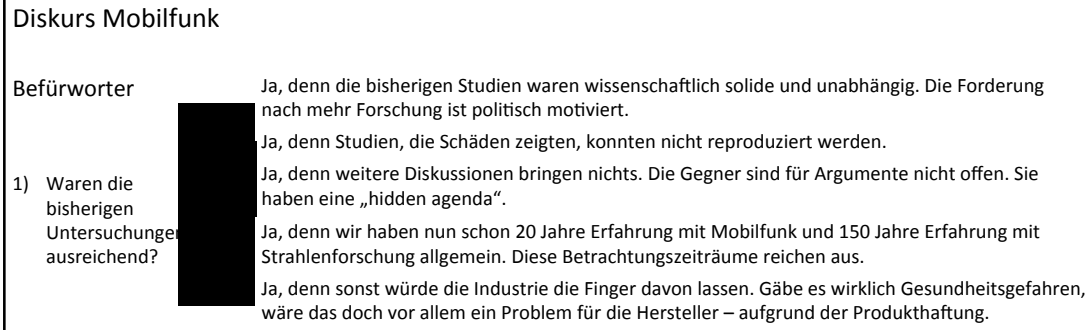

Abbildung 3: Mobilfunkdiskurs - Stimmen der Befürworter, wissenschaftlichtechnische Argumente

(Quelle: Eigene Darstellung)

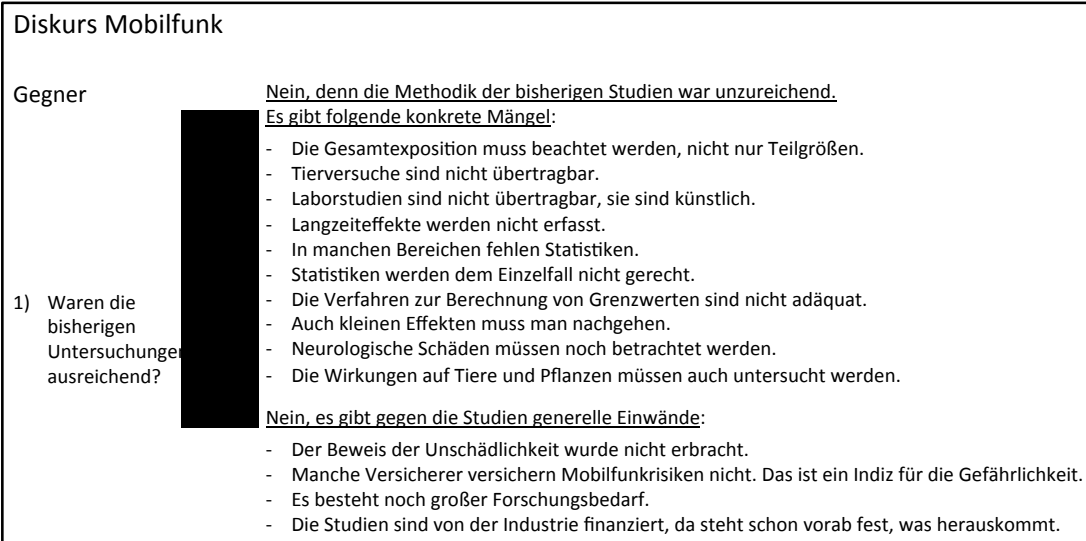

Abbildung 4: Mobilfunkdiskurs - Stimmen der Gegner, wissenschaftlich-technische Argumente

(Quelle: Eigene Darstellung)

(2) Stimmt es, dass wirklich nichts gefunden wurde? Auf diese Frage antworten die Befürworter mit einem herzhaften „Ja“. Damit ist die Sache aber nicht abgeschlossen, wie Abbildung 4 zeigt. Die Gegner haben mehrere Einwände, erinnern an bestimmte Studien, die bestimmte Schäden gezeigt hätten. Die Befürworter leugnen entweder diese Schäden oder bagatellisieren sie (,Keiner kann beurteilen, wie schlimm jetzt ein paar Kopfschmerzpatienten mehr sind", sagte ein Interviewpartner), oder sie verweisen darauf, dass diese Schäden nicht der Technik selbst vorzuwerfen seien, sondern dem Gebrauch, der von ihr gemacht wird (zum Beispiel, wenn Mobiltelefone beim Autofahren genutzt werden, wodurch die Unfallgefahr steigt). Eine bedeutende Antwortstrategie ist die Unterscheidung von Nachweisen und Hinweisen, auf die ich im Folgenden näher eingehen 
will. Ein Hinweis ist nur ein Indiz, ein Anzeichen, das nicht zwingend ist. Ein Nachweis hingegen ist ein kompletter Beweis des infrage stehenden Sachverhalts.

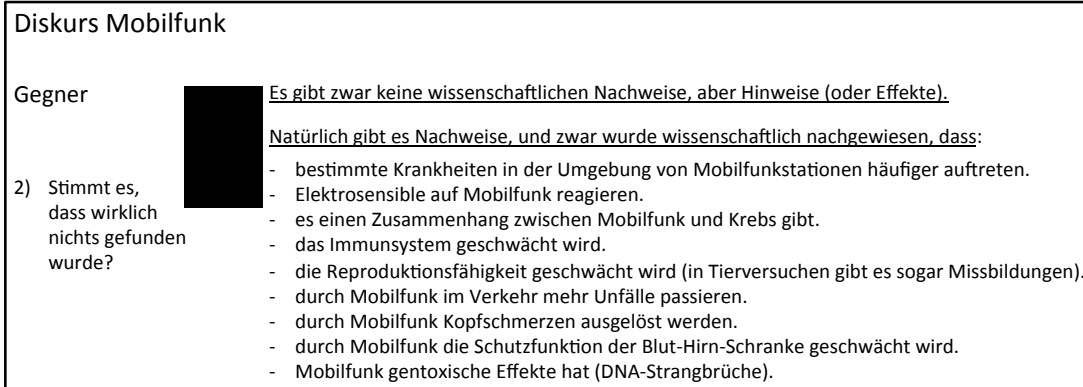

Abbildung 5: Mobilfunkdiskurs - Stimmen der Gegner, Interpretation der Resultate (Quelle: Eigene Darstellung)

Oft wurde zum Beispiel gesagt, es gebe zwar keine Nachweise von Schäden, wohl aber Hinweise. Taugt die Unterscheidung zwischen Hinweisen und Nachweisen im Mobilfunkdiskurs eher zur Unterstützung der Sache der Befürworter oder eher zur Unterstützung der Sache der Gegner? Es scheint, wie Karen Kastenhofer (in diesem Band) vermutet, dass die Vokabel der Nachweise eher zu dem Weltbild derjenigen gehört, die von einem beschränkbaren Erfahrungsraum ausgehen, während Hinweise eher zu einem nicht schließbaren Erfahrungsraum passen.

Etwas anders scheint es mit der Unterscheidung von Schäden und Effekten bestellt zu sein. Auch diese Unterscheidung wird eingesetzt, um den Dissens darüber, ob sich etwas nachweisen lässt oder nicht, auf eine neue Stufe zu heben. Es lässt sich manches finden, kann man mit dieser Unterscheidung sagen, aber das sind zunächst noch keine Schäden, sondern Effekte. Bei dieser Unterscheidung scheint es jedoch so zu sein, dass sie eher von den Befürwortern verwendet wird, und zwar zur Verteidigung gegen die Gegner. So dient sie insbesondere als Antwort auf die Feststellung, dass sich bei Leuten, die Mobilfunkstrahlung ausgesetzt sind, durchaus physiologische Effekte nachweisen lassen. Nun ja, kann dann der Befürworter sagen, es ist aber nicht jeder Effekt auch ein Schaden. Dennoch ist diese Unterscheidung auch vom Gegner zu verwenden, der sagen kann (und sagen wird), dass sich jeder Effekt über längere Zeit zu einem Schaden summieren kann.

So weit zu den Unterscheidungen. 
(3) Sollten wir den Stand der Dinge akzeptieren, auch wenn er keine definitive Sicherheit bietet? Die Frage, ob die Umstände so beschaffen sind, dass man das argumentum ad ignorantiam, auch wenn es keine definitive Sicherheit bietet, dennoch gelten lassen sollte, öffnet das Spektrum der moralisch-politischen Argumentationen. Hier geht es, wie in vielen gesellschaftlichen Diskussionen, um Nutzen und um (möglichen) Schaden. Und hier lassen sich, wie die Abbildungen auch deutlich machen, natürlich viele Pluspunkte für die Mobiltelefonie namhaft machen. Nicht nur die üblichen ökonomischen Gründe sprechen für die Technologie, nicht nur die Tatsache, dass die Konsumenten in ihr einen Nutzen zu finden vermögen. Vielmehr lässt sich eine überzeugende Verbindung zwischen dieser Technologie und dem Thema ,Rettung aus der Not' herstellen - denn durch Mobiltelefone werden beispielsweise die Anfahrtszeiten von Rettungsfahrzeugen nachweislich deutlich verkürzt. Schließlich verstärkt die Technologie auch das Sicherheitsgefühl gerade schwächerer Bevölkerungsgruppen.

Neben Nutzen und möglichem Schaden geht es bei den moralischpolitischen Argumenten auch darum, wer Nutznießer und wer potenziell Geschädigter ist. Diese Frage ist zentral, und es zeigt sich auch hier wieder, dass unter dem Titel des Risikos oft Gerechtigkeitsfragen verhandelt werden beziehungsweise dass Risikofragen ihre Brisanz gerade daraus beziehen, dass in ihnen Gerechtigkeitsfragen verborgen sind. Denn wenn, wie so oft, von Chancen und Risiken gesprochen wird, ist die Unterstellung, dass es ,unsere' Chancen und ,unsere' Risiken sind, häufig ungenau. Diejenigen, die bei neuen Risiken von den Chancen profitieren, sind nicht selten von denen verschieden, die mit den möglichen Schäden zurechtzukommen haben - darauf werden wir bei der Diskussion des Risikodiskurses über die Grüne Gentechnik noch zurückkommen.

Im Falle des Mobilfunks ist in unserem empirischen Material auffällig, dass nirgends die an sich mögliche kritische Position vertreten wird, dass nur wenige Profiteure, ihre' Technologie auf Kosten der Allgemeinheit durchdrücken möchten. Vielmehr scheint es so zu sein, dass breit akzeptiert wird, dass die Technologie vielen Parteien Nutzen bringt.

Hinzufügen könnte man, dass bei der Mobilfunktechnologie diejenigen, die den Nutzen haben, mit den potenziell Geschädigten weitgehend identisch sind. Denn aufgrund der Struktur der Technologie entsteht die bei Weitem höchste Strahlenbelastung nicht durch die Basisstationen, sondern an den Endgeräten. Insofern ist tatsächlich derjenige, der diese Technologie nutzt, auch derjenige, der den möglicherweise schädigenden Effekten am stärksten ausgesetzt ist. Also eine weitgehend symmetrische Situation. 
Freilich bleibt die - wenn auch wohl kleine - Gruppe derjenigen, die kein Mobiltelefon nutzen und dennoch den Strahlen der Basisstationen, dem Wireless LAN und den DECT-Telefonen der Nachbarn ausgesetzt sind.

$\mathrm{Zu}$ den relevanten Umständen gehört schließlich auch noch die Eigenschaft, dass die Technologie als solche reversibel ist - sie kann sozusagen, zumindest technisch gesehen, zurückgerufen werden, was typisch für eine elektronische Technik ist und sie in einer für Risikodiskurse relevanten Art und Weise von biologisch-chemischen Technologien unterscheidet, die meist nicht reversibel sind, weil sich Atome und Moleküle nicht mehr einsammeln lassen. Es mag eine Illusion sein, dass die Rückholung der Mobilfunktechnologie auch ökonomisch und politisch möglich ist, gleichwohl ist es denkbar, dass alle Handys eingezogen und alle Basisstationen abgebaut werden, sollte sich eines Tages doch herausstellen, dass die Strahlung gesundheitlich gefährlicher als gedacht ist.

\section{Rekonstruktion des Risikodiskurses Grüne Gentechnik: Zentrale Wortwechsel und Argumentationsdynamik}

Mehrere Interpreten meinen, eine zentrale Rolle ideologischer Streitpunkte im Gentechnikdiskurs erblicken zu können, und ziehen in Zweifel, dass es in der Auseinandersetzung um die Gentechnik tatsächlich (noch) um Risiken gehe. ${ }^{23}$ Mir erscheint demgegenüber durchaus vertretbar, diesen Diskurs wie auch den über Mobilfunk als Risikodiskurs zu beschreiben. Das ergibt sich schon daraus, dass das argumentum ad ignorantiam in diesem Diskurs eine prominente Rolle innehat. Der Risikodiskurs über die Grüne Gentechnik ist insoweit komplizierter als der Diskurs über Mobilfunk, als hier nicht nur mögliche Gesundheitsgefahren diskutiert werden, sondern auch ökologische Schäden. Gleichwohl lassen sich auch hier die vielfältigen Stimmen, die wir eingefangen haben, in übersichtlicher Weise an das argumentum ad ignorantiam anknüpfen, und auch die drei Fragen, welche den Finger auf die schwachen Punkte des Arguments legen, können entsprechend wieder die Spektren der konkreten Argumente eröffnen.

23 Besonders deutlich formuliert Wolfgang van den Daele (2001: 27): „Die Motive des Konflikts über die Gentechnik liegen in der Angst vor Unbekanntem, in moralischem Widerstand gegen das Verhältnis zur Natur, das durch die modernen Biotechnologien hergestellt wird, und in politischem Protest gegen die Macht und das Mandat der privaten Wirtschaft, die Gesellschaft mit technischen Innovationen und dem dadurch bedingten sozialen Wandel zu überziehen - und all das tendenziell in globalem Maßstab.“ 


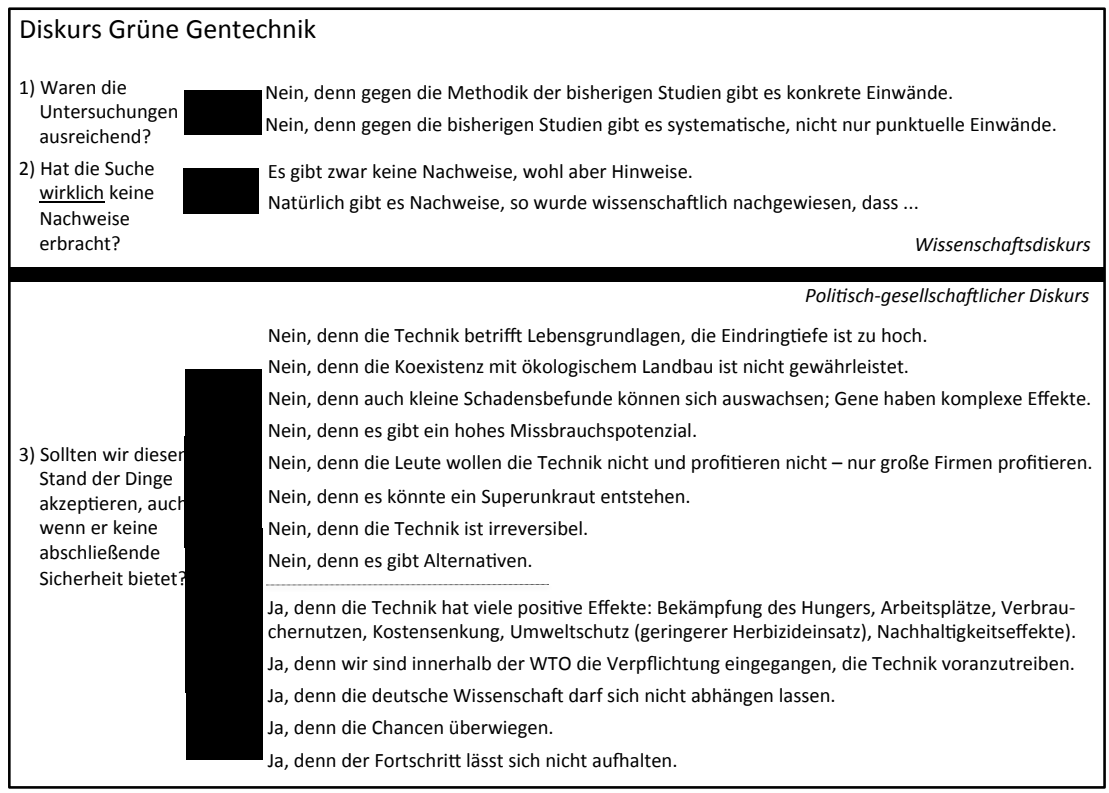

Abbildung 6: Der Wortwechsel im Diskurs über Grüne Gentechnik (Quelle: Eigene Darstellung)

(1) Waren die Untersuchungen ausreichend? Die Thematik der SchlieBung des Erfahrungsraumes ist im Falle der Grünen Gentechnik natürlich wesentlich virulenter als im Falle des Mobilfunks, da es hier nicht nur um mögliche gesundheitliche Auswirkungen geht, sondern auch um mögliche Umwelteffekte. Und die können potenziell alle Organismen betreffen, neben anderen Pflanzen auch Insekten, Vögel oder Säugetiere sowie andererseits auch Mikroorganismen und Pilze. Das scheint die Sache der Befürworter fast aussichtslos zu machen.

Immerhin hat die ökologische Schadensvermutung einen konkreten Anhaltspunkt: die Auskreuzung. Gentechnisch veränderte Pflanzen können über ihren Pollen ihr verwandeltes Erbgut auch auf andere Pflanzen übertragen. Ist dies ein Schaden? Es kann zum Beispiel ein ganz handfester ökonomischer Schaden sein, wenn diese anderen Pflanzen zu dem Acker eines ökologisch wirtschaftenden Nachbarbauern gehören, der daraufhin seine Ernte nicht mehr als ökologisch erzeugt absetzen kann und konkrete finanzielle Einbußen hat. Nach dem von der damaligen rot-grünen Regierungskoalition in Deutschland beschlossenen Haftungsrecht ist dies sogar für den Bauern, der die GMOs gesät hat, ein handfester Schaden, da er für den finanziellen Verlust seines Nachbarn haftbar gemacht werden kann. 
Kreuzen die gentechnisch veränderten Pflanzen jedoch auf Wildpflanzen aus, so hat zunächst einmal niemand einen unmittelbaren Schaden davon. Es ist aber, sagen die Gegner, zu beachten, dass die Gene ja ganz besondere Eigenschaften transportieren, zum Beispiel Resistenz gegen Unkrautvernichtungsmittel oder Resistenz gegen bestimmte Schädlinge. Kreuzen nun auch diese Eigenschaften auf Wildpflanzen aus, so könnte es sein, dass sehr robuste Pflanzen das Ergebnis sind, ähnlich wie jene Ratten, die in „Asterix und Obelix in Rom“ vom Zaubertrank naschten und plötzlich ungeheuer stark wurden. Solche Pflanzen könnten sowohl auf dem Acker als Unkräuter Probleme machen wie auch in natürlichen Habitaten andere Arten verdrängen; sie stellen somit auch eine Gefährdung für die Biodiversität dar.

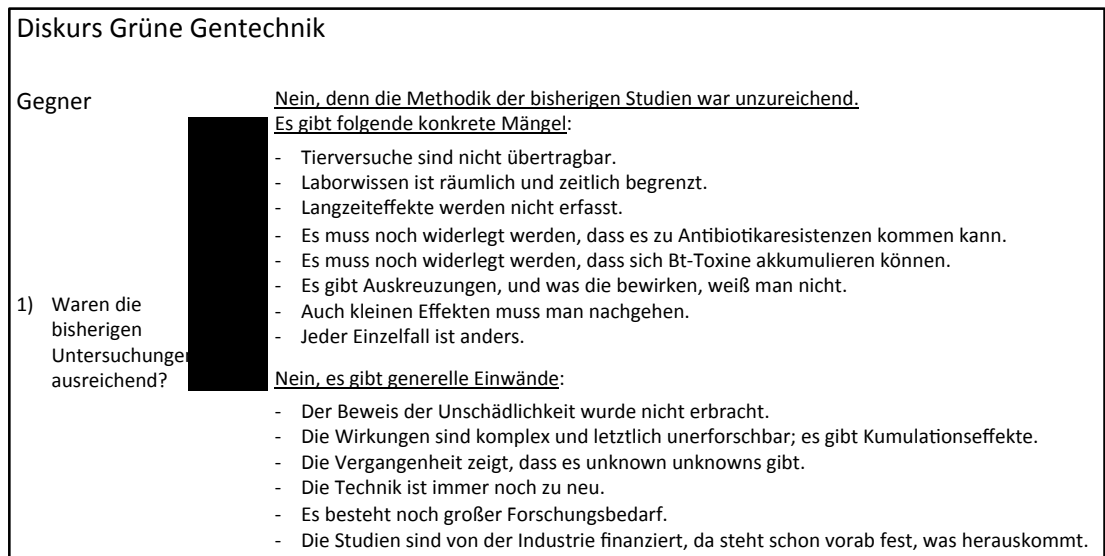

Abbildung 7: Diskurs Grüne Gentechnik - Stimmen der Gegner, wissenschaftlichtechnische Argumente

(Quelle: Eigene Darstellung)

Größer ist die Einigkeit, was als ein gesundheitlicher Schaden anzusehen ist, der durch den Konsum von Produkten entsteht, die mithilfe von oder aus gentechnisch veränderten Pflanzen hergestellt wurden. Der Streit geht dann um die Frage, ob solche Schäden nachgewiesen wurden oder nicht. Tatsächlich weisen die Befürworter darauf hin, dass die gentechnisch modifizierten Produkte wesentlich aufwendiger geprüft seien als die allermeisten herkömmlichen Produkte. Zum anderen seien da die vielen Millionen Nordamerikaner, die seit Jahren schon gentechnisch veränderten Mais und andere GMO-Produkte konsumieren - ohne dass bislang nachteilige gesundheitliche Effekte (die sich auf die genetische Modifikation zurückführen ließen) deutlich geworden seien. Hierzu merken jedoch die 
kritischen Stimmen unter anderem an, dass keineswegs überprüft worden sei, ob tatsächlich keine gesundheitlichen Schäden aufgetreten seien, da entsprechende Vergleichsstudien nie durchgeführt worden seien.

(2) Stimmt es, dass wirklich nichts gefunden wurde? In diesen beiden Bereichen, den möglichen ökologischen Schäden und den Gesundheitsschäden, lassen sich die beiden Fragen oft nicht genau trennen beziehungsweise die Stimmen lassen sich meist sowohl dem einen Aspekt wie auch dem anderen zuordnen. Oft ist hier die Frage, wie ein Befund zu interpretieren sei, verbunden mit dem Hinweis, dass etwas Bestimmtes noch gar nicht untersucht worden ist:

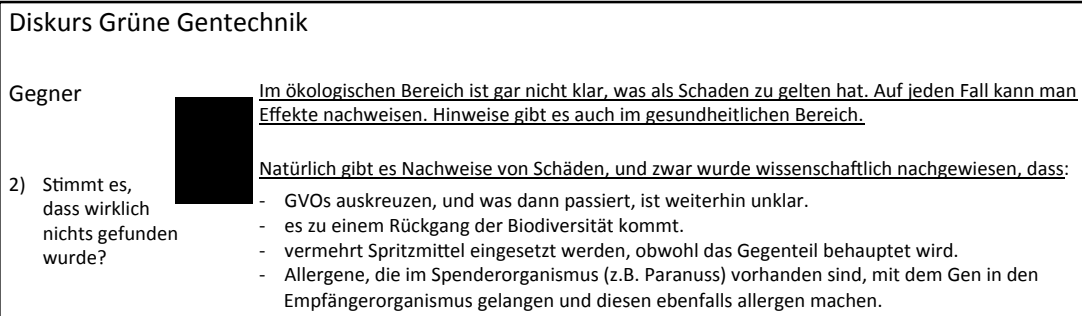

Abbildung 8: Diskurs Grüne Gentechnik - Stimmen der Gegner, Interpretation der Resultate

(Quelle: Eigene Darstellung)

Wie man sieht, ist auch in diesem Diskurs die Unterscheidung von Nachweisen und Hinweisen, auf die ich oben bereits einging, wichtig. Auch die etwas anders gelagerte Unterscheidung von Effekten und Schäden findet man. Und hieran knüpft sich erneut ein Streit, wie man beides unterscheiden soll - ein Streit, der besonders im ökologischen Bereich nicht leicht zu entscheiden ist. Auskreuzung wird aber auch ganz unabhängig von solchen konkreten Schadenshypothesen von vielen Gegnern als etwas sicher Unerwünschtes angenommen. Dies ist nur verständlich vor dem Hintergrund der kontroversen Beurteilung der Gentechnik als solcher. Für die einen ist sie eine Weiterentwicklung und Verfeinerung der klassischen Züchtung. Für die anderen ist sie etwas völlig Andersartiges, und zwar insbesondere, weil Gentechnik es gestattet, artübergreifend zu züchten, also etwa ein Bakteriengen in eine Kulturpflanze einzusetzen. Die Produkte der Gentechnik sind demnach für die einen verbesserte Züchtungen, für die anderen Monstren. Und entsprechend bedeutet auch Auskreuzung für die einen etwas wenig Aufregendes, für die anderen aber so etwas wie eine Verseuchung der Natur, das Einsickern monströser Objekte in einen 
zuvor unberührten Bereich. Und das wäre dann sehr wohl ein massiver Schaden.

Auskreuzung kann zwar durch Sicherheitszonen und Auswahl der Pflanzensorten gesteuert werden, ausgeschlossen werden kann sie kaum. Wir haben hier also die Besonderheit, dass ein bestimmtes Ereignis von Befürwortern wie Gegnern als möglich oder sogar als wahrscheinlich angesehen wird, dass aber dieses Ereignis verschieden bewertet wird. Für die Befürworter ist es weiter nichts Bedenkliches, für die Gegner ein massiver Schaden.

Diese Divergenz kann nur verstanden werden, wenn man auf den dahinterliegenden (normativen) Naturbegriff zurückgeht. Wer Natur als das Andere zur Menschenwelt konzipiert, wie es in der Tradition auch üblich war, oder gar als Schöpfung Gottes, für den muss das Einsickern menschgemachter Erbanlagen in den natürlichen Genpool bedenklich sein. Schon die Technologie selbst muss von einem solchen Ausgangspunkt höchst bedenklich erscheinen, und ihr Unterschied zur herkömmlichen Züchtung erscheint in dieser Perspektive markant. Wer hingegen davon ausgeht, dass es eine ,unberührte Natur' auf unserem Planeten nicht mehr gibt, auBer vielleicht an manchen Orten in der Tiefsee, wer der Meinung ist, dass die Natur, die uns umgibt, wenn wir im Wald spazieren gehen, aufs Land fahren oder eine Exkursion in ein Naturschutzgebiet machen, von vornherein schon innerlich, nicht nur äußerlich, von Menschen beeinflusst ist, für den ist die Freisetzung gentechnisch veränderten Erbguts nicht von vornherein ein großer Schaden. In dieser Bedeutung unterschiedlicher Naturbegriffe (und damit verbunden unterschiedlicher Technikbegriffe) liegt das, was manche Interpreten als das ideologische Moment an der Debatte bezeichnen. Schon die Tatsache, dass Fragen der Bewertung im Gentechnikdiskurs eine solche Rolle einnehmen, weist auf darunterliegende Kontroversen hin, die diesen Diskurs von dem zuvor diskutierten über Mobilfunk unterscheiden.

Schließlich gibt es auch in diesem Diskurs einige divergente Interpretationen von Studienergebnissen, die den vielfach belegten Kontroversen im Mobilfunkdiskurs entsprechen. So wird etwa von denen, die behaupten, dass sehr wohl gesundheitliche Schäden durch den Konsum gentechnisch veränderter Lebensmittel nachgewiesen werden können, immer wieder die Studie von Arpad Pusztai aus dem Jahr 1995 angeführt. Pusztai hatte Kartoffeln, denen ein Gen von Schneeglöckchen eingeführt worden war, an Ratten verfüttert und deutliche Schädigungen nachweisen können. Jedoch wurden seine Studien in der Folge angezweifelt. Nicht angezweifelt wurde hingegen eine Studie zu transgenen Erbsen, die 2005 im australischen 
Canberra durchgeführt wurde und die in Fütterungsversuchen mit Mäusen deutliche Schädigungen nachweisen konnte. Die Versuche zu der neuen transgenen Erbsensorte wurden jedoch eingestellt, womit der Fall für die Befürworter zum Exempel eines funktionierenden Kontrollsystems wurde. Einigkeit besteht weiterhin darin, dass Allergene übertragen werden können - und dass dies kontrolliert werden muss.

(3) Sind die Umstände so beschaffen, dass man diesen Stand der Dinge akzeptieren sollte, auch wenn er keine definitive Sicherheit bietet? Gerade bei der Grünen Gentechnik bleibt ein erhebliches Besorgnispotenzial bei den Gegnern. Wie gehen die Befürworter damit um? Auch hier gibt es wieder verschiedene Strategien. Die eine ist die Verkleinerung der Risiken durch Vergleich. ${ }^{24}$ So wird darauf hingewiesen, dass die vorgetragenen Risikohypothesen, die gentechnisch veränderten Pflanzen zugewiesen werden, genauso oder noch mehr auch für konventionelle und ebenso auch für Pflanzen aus ökologischem Anbau gelten. Diese Hinweise könnten überzeugend sein, allerdings werden von den Gegnern andere Aspekte ins Feld geführt, zum einen die mangelnde Reversibilität der Technik: Während Mobilfunk theoretisch zurückholbar wäre, gilt das für gentechnisch veränderte Pflanzen wohl nicht.

Das ist das eine. Das andere ist die Frage nach dem möglichen Nutzen. Die Befürworter führen hier viele Punkte ins Feld, die für sich genommen eigentlich ungewöhnlich überzeugend klingen sollten. GMOs könnten plausibel in Anspruch nehmen, etwas zur Nachhaltigkeit beizutragen, weil weniger Pestizide eingesetzt werden müssten, sie lieferten höhere Erträge und könnten daher auch möglicherweise einen Beitrag zur Minderung des Hungers in der Welt liefern. Allerdings werden einerseits die Belege, die dafür geliefert werden, vielfach bestritten, und teilweise auch durch Gegenstudien widerlegt, andererseits wirken sie auf die Gegner auch als ÄuBerung der Nahrungsmittelindustrie wenig glaubhaft.

Auffällig ist aber, dass es nicht zu gelingen scheint, darzulegen, wo der konkrete Nutzen für den individuellen Verbraucher liegen soll. GMOs schmecken nicht besser, sie sind nicht billiger und die Eigenschaften, die

24 Die Verkleinerung durch Vergleich ist eine alte rhetorische Technik, die ausführlich etwa bei Quintilian (Ausbildung des Redners: 2. Teil, Buch VIII, Kap. 4) beschrieben wird. Quintilian zeigt auch, dass der Vergleich ebenso zum Vergrößern taugt, was in Risikodiskursen, auch in den besprochenen, ebenfalls häufig vorkommt, wenn etwa darauf verwiesen wird, dass auch FCKW als sicher galten - bis deren Langzeiteffekte in der Stratosphäre bekannt wurden. Wolfgang van den Daele spricht in Bezug auf den Gentechnikdiskurs ähnlich von einer „Normalisierung der Risiken durch Vergleich“ (van den Daele 2001: 41f.). 
ihnen die Gentechnik angedeihen lässt, haben in aller Regel den Zweck, die Produktion und/oder die Verarbeitung dieser Nutzpflanzen zu optimieren. Davon hat aber nur der Produzent oder der Fabrikant etwas, nicht aber der Verbraucher. Und hier haben die Befürworter mit einem Problem zu kämpfen, denn während der Verbraucher keinen Nutzen hat, ist doch er es, der mit dem möglichen ökologischen oder gesundheitlichen Schaden im Falle eines Falles zurechtkommen müsste. Es liegt hier also der Verdacht nahe (und wird auch ausgesprochen), dass der Nutzen privatisiert wird, während der mögliche Schaden auf die Allgemeinheit abgewälzt wird. ${ }^{25}$

Diese Asymmetrie unterscheidet meiner Ansicht nach den Diskurs über GMOs markant von dem Diskurs über Mobilfunk. Sie dürfte einer der Gründe dafür sein, dass Grüne Gentechnik vor allem in Europa ein negativeres Image hat als die Mobilfunktechnologie. Denn weil die Nutznießer der Grünen Gentechnik vor allem die Agroindustrie und die Saatguthersteller sind, lässt sich diese Technologie viel leichter als Machenschaft ,der Industrie' ansehen. Das ist zwar prinzipiell auch beim Mobilfunk möglich, hier jedoch nur um den Preis einer gewissen Zwiespältigkeit, da dieselben Menschen, die fröhlich ins Handy oder in ihr Schnurlostelefon sprechen, auch diejenigen sind, die gegen die Basisstation gegenüber ihrer Wohnung protestieren. Hierin kommt natürlich eine deutliche Verdrängung ins Spiel, die in manchen Protesten unübersehbar ist.

Allerdings ist auch hier das letzte Wort noch nicht gesprochen. Denn die Firmen arbeiten bekanntlich schon an der zweiten Generation gentechnisch modifizierter Pflanzen. Sie planen Pflanzen, die dem Verbraucher einen konkreten Nutzen bringen sollen, wie etwa verbesserte Vitaminversorgung oder sogar nikotinfreie Tabakpflanzen. Ob allerdings solche Pflanzen der Debatte, nachdem sie einmal die beschriebenen Bahnen genommen hat, eine neue Richtung geben werden, bleibt abzuwarten.

25 Die Bedeutung der sozialen Verteilung von Nutzen und Lasten für die Akzeptanz von Technologien ist in der Risikoforschung seit Langem bekannt, siehe nur die Bemerkung von Ortwin Renn und Michael Zwick (1997: 3): „Die Öffentlichkeit hat im Umgang mit Risiken Heuristiken entwickelt, die auf qualitativen Risikomerkmalen beruhen (...): das Katastrophenpotential, die soziale Verteilung von Nutzen und Lasten risikobehafteter Technologien, ihre Kontrollierbarkeit und die wahrgenommene Freiwilligkeit der Risikoübernahme.“ Das sind die Grundannahmen der psychometrischen Risikoforschung. 


\section{Fazit}

Das vorgestellte Modell, das in den vorstehenden Abschnitten beschrieben wurde, darf nicht mit dem Diskurs selbst verwechselt werden. Der Diskurs selbst (zu einer bestimmten Zeit) ist nach der oben erläuterten Definition die Gesamtheit aller (institutionellen und individuellen) Stimmen, die sich öffentlich zum Thema Risiken von Mobilfunk (bzw. Grüner Gentechnik) geäußert haben und sich dabei explizit oder implizit auf andere Stimmen beziehen. All diese Stimmen einzeln zu hören und zu bewerten, übersteigt die Möglichkeiten jedes endlichen Hörers und Lesers. Umso wichtiger ist ein guter Ausgangspunkt für eine differenzierte Auseinandersetzung mit diesen Stimmen. Man sieht anhand dieses Modells etwas Neues, kann mögliche Argumentationslinien ausfindig machen, solche also, die verfolgt werden könnten (auch wenn sie bisher von niemandem beschritten wurden), man kann aber auch tote Geleise identifizieren. Das Modell verdeutlicht die Struktur von Diskursen unter Nichtwissensbedingungen.

Das Modell kann nun auf verschiedene Weise genutzt werden. Zum einen kann es der eigenen Meinungsbildung und Meinungsreifung dienen. Dabei helfen würde auch eine elektronische Version, die bei den geäußerten Argumenten den Nutzer fragen würde, ob und welches Gegenargument er dazu vorbringen würde. So könnte eine bereits antike Anweisung der Sophisten neu umgesetzt werden, die vom guten Redner verlangt, gleich kompetent für wie gegen eine Sache argumentieren zu können. Zumindest ist es auch für denjenigen, der sich schon eine Meinung gebildet hat, sinnvoll, sich durch ein solches Instrument auch mit den Ansichten der Gegenseite präzise auseinanderzusetzen.

Insgesamt weist die Rekonstruktion darauf hin, welche Möglichkeiten es gibt, in Situationen, in denen negative Evidenz behauptet wird, konkrete und sinnvolle Rückfragen zu stellen, um so die Risikoforschung insgesamt oder im konkreten Fall weiterzuentwickeln. Sie zeigt aber auch, dass es grundlegende Aporien gibt, die jeder Form von Risikoforschung anhaften und die sich nicht auflösen lassen. Hierzu zählt die Divergenz zwischen dem Zeithorizont einer sachlich angemessenen Risikoforschung, der eher in Jahrzehnten auszudrücken wäre, und dem Zeithorizont der Wirtschaft, der eher nach Monaten zählt. Hierzu zählt auch die Unmöglichkeit, die unendlich vielen Kombinationen von Faktoren, die in der wirklichen Welt zusammenwirken, um (unerwünschte) Wirkungen hervorzubringen, durch endlich viele Kombinationen im Labor erschöpfend zu überprüfen. Es sind gerade auch diese Aporien, die immer wieder nahelegen, auf die normative Ebene zu wechseln. 
Das Arrangement der Stimmen kann ferner auch verwendet werden, um eine Voraussage über die weitere Entwicklung des Diskurses zu treffen, gerade weil das Arrangement auch etwas von der internen Logik des Diskurses erkennen lässt. Das Arrangement erleichtert das „balancing of consideration", das schon Walton (1996: 285) forderte, um ein Nichtwissensargument einschätzen zu können.

So wäre etwa meine Einschätzung des Mobilfunkdiskurses, dass dieser sich, wenn sich nicht entscheidende, deutliche Schadensnachweise ergeben, abschwächen wird, und dass die Mobilfunktechnologie sich immer weiter ausbreiten wird (wodurch übrigens der Nachweis von Schäden immer schwieriger werden wird, da ,unbelastete Kontrollgruppen' fehlen werden), auch wenn es zu keiner anderen Technologie mehr Bürgerinitiativen in Deutschland gibt - insgesamt über 10.000. Denn in diesem Diskurs scheint mir die gefundene Evidenz für Schäden insgesamt zu niedrig zu sein (das Phänomen der Elektrosensibilität). Andererseits bringt die Mobilfunktechnologie in der Wahrnehmung der Mehrheit erhebliche und sehr vielfältige Vorteile. Diese Vorteile kommen sehr unterschiedlichen, nicht nur einzelnen Bevölkerungsgruppen zugute. Sie sind breit verankert. Und was die möglichen Nachteile angeht, so sind diese normalerweise vor allem abhängig vom individuellen Verhalten des Nutzers, da die höchste Strahlenexposition an den Endgeräten entsteht. Insofern ist es hier normalerweise dieselbe Person, die den Nutzen und den möglichen Schaden hat - und diese Person kann sogar das Ausmaß des möglichen Schadens durch ihr individuelles Verhalten steuern. Hinsichtlich der Distribution von Nutzen und (möglichem) Schaden ist also eine Symmetrie zu beobachten, ein Zustand, der gemeinhin als gerecht angesehen wird. Bei dieser Konstellation ist langfristig zu erwarten, dass die möglichen Risiken akzeptiert werden, auch wenn einzelne Gruppen (vor allem die Elektrosensiblen) weiter opponieren werden. Zugleich kann vonseiten der Befürworter immer auch argumentiert werden, dass die Technik, sollten sich doch einmal wirkliche Schäden zeigen, jederzeit wieder zurückgerufen werden kann. Sie ist, zumindest theoretisch, reversibel.

Anders sieht es im Falle der Grünen Gentechnik aus. Hier gibt es zwar nur schwache Hinweise auf gesundheitliche Schäden, und auch die Risikohypothesen, die sich um die möglichen ökologischen Schäden ranken, sind umstritten. Es könnte sein, dass der eigentliche Motor des Diskurses nicht hier liegt, sondern eher im ,unteren Teil' des Arrangements, bei den moralisch-politischen Einwänden. Denn die klassische Frage, wem die Technologie nutzt und wer von möglichen Schäden betroffen wäre, lässt sich eindeutig beantworten. Und diejenigen, die den Nutzen haben, sind 
nicht identisch mit denjenigen, die mit dem möglichen Schaden zu kämpfen haben werden. Es ist im Bereich der Grünen Gentechnik nicht gelungen, den Konsumenten überzeugend zu vermitteln, dass sie einen Nutzen von dieser Technologie haben werden.

Bei öffentlich geförderten Projekten der Risikoforschung und des Risikodialogs wird oft die bereits erwähnte Formel von den Chancen und Risiken verwandt. Suggeriert wird dabei, dass es objektive Chancen und objektive Risiken gebe und dass es nur darum gehe, nachzusehen, ob die einen die anderen überwiegen, beziehungsweise darum, die Risiken einzudämmen, damit das Resultat akzeptabel aussieht. Mit dieser Formel wird aber das eigentliche Problem bei Risikodiskursen verdeckt, gerade indem es objektiviert wird. Denn das Kritische an riskanten Technologien ist nicht, dass es überhaupt Risiken gibt - und nicht nur Chancen -, sondern dass diejenigen, die die Chancen nutzen wollen, in der Regel von denen verschieden sind, die die Risiken tragen sollen.

Bezogen auf die Grüne Gentechnik bedeutet das: Gentechnisch veränderte Tomaten (um ein Beispiel zu wählen) schmecken (bislang) nicht besser, sie sind auch nicht billiger. Das Argument, dass auf diese Weise der Hunger in der Welt gelindert werden könnte, wirkt aus dem Munde von Vertretern der Nahrungsmittelindustrie eher wenig überzeugend, ebenso wenig wie die Behauptung, durch gentechnisch veränderte Pflanzen könne der Pestizideinsatz verringert werden. Der Diskurs speist sich hier aus der Differenz von Nutznießern und potenziell Geschädigten, während der Streit über den Mobilfunk sich gerade aus der Identität von Nutznießern und potenziell Geschädigten zu befrieden scheint. Zugleich spielt im Gentechnikdiskurs auch ein normativer Naturbegriff eine erhebliche Rolle; die Technologie als solche wird vielfach abgelehnt, weil sie für viele eine Grenze des Machbaren überschreitet. Überdies ist die Grüne Gentechnik im Gegensatz zum Mobilfunk nicht reversibel. Einmal eingesetzt, kann sie voraussichtlich kaum zurückgeholt werden. Während also die Mobilfunktechnologie meiner Einschätzung nach akzeptiert werden wird, ist mit einer Akzeptanz der Grünen Gentechnik in Europa vorerst nicht zu rechnen. ,Vorerst' bedeutet dabei auf eine Zeit von vielleicht zehn Jahren. Langfristig wird es vermutlich anders aussehen, vor allem dann, wenn um Europa herum überall gentechnisch modifizierte Organismen gepflanzt und geerntet werden.

Mögen diese abschließenden Einschätzungen nun überzeugen oder nicht - eines sollten sie in jedem Fall belegen: Die vorgeschlagene Strukturierung beider Risikodiskurse durch das argumentum ad ignorantiam 
ermöglicht nicht nur einen Überblick, sondern auch methodische Verglei-
che zwischen Risikodiskursen unter Bedingungen von Nichtwissen.

\section{Literatur}

Aristoteles: Rhetorik. Übersetzt, mit einer Bibliographie, Erläuterungen und einem Nachwort von Franz G. Sieveke. Paderborn: UTB Wilhelm Fink, 1989.

Aristoteles: Topik. Übersetzt und mit Anmerkungen versehen von Eugen Rolfes. Hamburg: Meiner, 1968.

Bachtin, Michail (1971/1963): Probleme der Poetik Dostoevskijs. München: Carl Hanser.

Baumgartner, Michael/Graßhoff, Gerd (2004): Kausalität und kausales Schließen. Eine Einführung mit interaktiven Übungen. Bern: Studies.

Böschen, Stefan/Kastenhofer, Karen/Rust, Ina/Soentgen, Jens/Wehling, Peter (2010): The Political Dynamics of Scientific Non-Knowledge. The Cases of Agro-Biotechnology and Mobile Phoning. In: Science, Technology \& Human Values 35, 783-811.

Cook, Guy (2004): Genetically Modified Language. London/New York: Routledge.

Copi, Irving M. (1968): Introduction to Logic. 3. Aufl., London: Macmillan \& Company.

Duncan, Ronald/Weston-Smith, Miranda (Hg.) (1977): Encyclopaedia of Ignorance. 2 Bde. Oxford et al.: Pergamon.

Gigerenzer, Gerd (2007): Bauchentscheidungen. Die Intelligenz des Unbewussten und die Macht der Intuition. Gütersloh: C. Bertelsmann.

Grasberger, Thomas/Kotteder, Franz (2003): Mobilfunk. Ein Freilandversuch am Menschen. München: Kunstmann.

Hajer, Maarten A. (2003): Argumentative Diskursanalyse. Auf der Suche nach Koalitionen, Praktiken und Bedeutung. In: Keller, Reiner/Hirseland, Andreas/Schneider, Werner/ Viehöver, Willy (Hg.): Handbuch Sozialwissenschaftliche Diskursanalyse, Bd. 2: Forschungspraxis. Opladen: Leske + Budrich.

Hansen, Hans V./Pinto, Robert C. (Hg.) (1995): Fallacies: Classical and Contemporary Readings. University Park, PA: Pennsylvania State University Press.

Jones, Timothy (1990): The Origins of Human Diet and Medicine. Tucson: University of Arizona Press.

Kastenhofer, Karen (2007): Technische Physiker zu EMF. Augsburg: Projekt „Nichtwissenskulturen" (unveröff. Ms.).

Keith, William/Rehg, William (2008): Argumentation in Science: The Cross-Fertilization of Argumentation Theory and Science Studies. In: Hackett, Edward J./Amsterdamska, Olga/Lynch, Michael/Wajcman, Judy (Hg.): The Handbook of Science and Technology Studies. 3. Aufl., Cambridge, MA: MIT Press, 211-239.

26 Mit anderer Gewichtung und wesentlich kürzer wurden die in diesem Text vorgestellten Ergebnisse unter dem Titel „Das argumentum ad ignorantiam als Schlüssel zu den Risikodiskursen über Grüne Gentechnik und Mobilfunk“ in der Zeitschrift für Diskursforschung, 2. Jg. (2014), Heft 2, 117-141 veröffentlicht. 
Kendrew, John (1977): Introduction. In: Duncan, Ronald/Weston-Smith, Miranda (Hg.): Encyclopaedia of Ignorance. 2 Bde. Oxford et al.: Pergamon, 205-207.

Koselleck, Reinhart (1995): „Erfahrungsraum“ und „Erwartungshorizont“ - zwei historische Kategorien. In: ders.: Vergangene Zukunft. Zur Semantik geschichtlicher Zeiten. Frankfurt a. M., 349-375.

Krabbe, Erik C. W. (1995): „Appeal to Ignorance“. In: Hansen, Hans V./Pinto, Robert C. (Hg.): Fallacies: Classical and Contemporary Readings. University Park, PA: Pennsylvania State University Press, 251-264.

Lassen, Jesper/Jamison, Andrew (2006): Genetic Technologies Meet the Public. In: Science, Technology \& Human Values 31, 8-26.

Locke, John (1962/1690): Über den menschlichen Verstand. Übersetzt von Carl Winckler, Bd. II, Berlin: Akademie-Verlag.

Martin, Andrew (1985): The Knowledge of Ignorance. From Genesis to Jules Verne, Cambridge University Press.

Perelman, Chaim/Olbrechts-Tyteca, Lucie (1999/1958): Traité de l'Argumentation. La Nouvelle Rhétorique. 2 Bde. Paris: Presses Universitaires de France.

Plinius Secundus: Naturalis Historiae liber xxix, XXIV (manchmal auch XXIII). (Ed. Karl Friedrich Theodor Mayhoff, Ludwig Jan), Bibliotheca Teubneriana, Leipzig 1897, diverse Nachdrucke.

Quintilian (Marcus Fabius Quintilianus): Ausbildung des Redners. Zwölf Bücher. Herausgegeben und übersetzt von Helmut Rahn. Zweiter Teil. Darmstadt: Wissenschaftliche Buchgesellschaft, 1995.

Renn, Ortwin/Zwick, Michael (1997): Risiko- und Technikakzeptanz. Berlin et al.: Springer.

Rescher, Nicholas (1999): The Limits of Science. Revised Edition. Pittsburgh: University of Pittsburgh Press.

ders. (2009): Ignorance. Pittsburgh: University of Pittsburgh Press.

Rust, Ina (2006b): Argumentationsanalyse Grüne Gentechnik. Augsburg: Projekt „Nichtwissenskulturen" (unveröff. Ms.).

dies. (2006a): Gestaltungsöffentlichkeit Mobilfunk. Kapitel 1: Argumentationsanalyse Darstellung des Materials. Augsburg: Projekt „Nichtwissenskulturen” (unveröff. Ms.).

Schiemann, Gregor (1997): Wahrheitsgewissheitsverlust. Hermann von Helmholtz' Mechanismus im Anbruch der Moderne. Eine Studie zum Übergang von klassischer zu moderner Naturphilosophie. Darmstadt: Wissenschaftliche Buchgesellschaft.

Spencer Brown, George (1971): Laws of Form. London: Allen and Unwin.

van den Daele, Wolfgang (2001): Besonderheiten der öffentlichen Diskussion über die Risiken transgener Pflanzen. Dynamik und Arena eines Modernisierungskonflikts. In: Münchner Rück (Hg.): 5. Internationales Haftpflicht-Forum. München: Münchner Rück, 24-89.

van den Daele, Wolfgang/Pühler, Alfred/Sukopp, Herbert (1997): Transgenic HerbicideResistant Crops. A Participatory Technology Assessment. WZB Discussion Paper FS II 97-302. Berlin: WZB.

van Eemeren, Frans H./Grootendorst, Rob (2004): A Systematic Theory of Argumentation. The Pragma-Dialectical Approach. Cambridge: Cambridge University Press.

Walton, Douglas N. (1996): Arguments from Ignorance. University Park, PA: Pennsylvania State University Press. 


\section{Jens Soentgen}

ders. (2006): Fundamentals of Critical Argumentation. Cambridge: Cambridge University Press.

Wehling, Peter (2006): Im Schatten des Wissens? Perspektiven der Soziologie des Nichtwissens. Konstanz: UVK.

Wiedemann, Peter M./Mertens, Johannes/Schütz, Holger/Hennings, Wilfried/Kallfass, Monika (2001): Risikopotentiale elektromagnetischer Felder: Bewertungsansätze und Vorsorgeoptionen. Band 1. Jülich: Forschungszentrum Jülich.

Wiedemann, Peter M./Schütz, Holger/Spangenberg, Albena (2005): Bewertung der wissenschaftlichen Literatur zu den Risikopotenzialen von hochfrequenten elektromagnetischen Feldern des Mobilfunks. Teil 1: Darstellung und Diskussion der Themenfelder. Jülich: Forschungszentrum Jülich.

Wiedemann, Peter M./Schütz, Holger/Thalmann, Andrea T. (2003): Mobilfunk und Gesundheit. Risikobewertung im wissenschaftlichen Dialog. Jülich: Forschungszentrum Jülich. 